\title{
Investigation of the Chemical Changes from Crude and Processed Paeoniae Radix Alba-Atractylodis Macrocephalae Rhizoma Herbal Pair Extracts by Using Q Exactive High-Performance Benchtop Quadrupole-Orbitrap LC-MS/MS
}

\author{
Gang Cao, ${ }^{1,2}$ Qinglin Li, ${ }^{3}$ Hao Cai, ${ }^{1}$ Sicong Tu, ${ }^{4}$ and Baochang Cai ${ }^{1,2}$ \\ ${ }^{1}$ College of Pharmacy, Nanjing University of Chinese Medicine, Nanjing 210023, China \\ ${ }^{2}$ Research Center of TCM Processing Technology, Zhejiang Chinese Medical University, Hangzhou 310053, China \\ ${ }^{3}$ Zhejiang Cancer Hospital, Hangzhou 310022, China \\ ${ }^{4}$ Faculty of Medicine, University of New South Wales, Sydney, NSW 2031, Australia \\ Correspondence should be addressed to Hao Cai; haocai_98@126.com
}

Received 20 January 2014; Revised 7 March 2014; Accepted 20 March 2014; Published 4 May 2014

Academic Editor: Shi-Biao Wu

Copyright (c) 2014 Gang Cao et al. This is an open access article distributed under the Creative Commons Attribution License, which permits unrestricted use, distribution, and reproduction in any medium, provided the original work is properly cited.

\begin{abstract}
The Paeoniae Radix Alba-Atractylodis Macrocephalae Rhizoma herbal pair is mainly used for regulating the functions of liver and spleen, benefiting $q i$, and nourishing blood. However, the bioactive compounds for the pharmacological activities of the crude and processed Paeoniae Radix Alba-Atractylodis Macrocephalae Rhizoma herbal pair extracts are still unclear to date. In the present study, Q Exactive high-performance benchtop quadrupole-Orbitrap LC-MS/MS was applied to identify the complicated components from crude and processed Paeoniae Radix Alba, crude and processed Atractylodis Macrocephalae Rhizoma, and their crude and processed herbal pair extracts. 123 and 101 compounds were identified in crude and processed Paeoniae Radix Alba samples, respectively. Meanwhile, 32 and 26 compounds were identified in crude and processed Atractylodis Macrocephalae Rhizoma samples, respectively. In the crude and processed Paeoniae Radix Alba-Atractylodis Macrocephalae Rhizoma herbal pair extracts, co-decoction could significantly change the chemical composition of Paeoniae Radix Alba and Atractylodis Macrocephalae Rhizoma in solution. The developed method may provide a scientific foundation for deeply elucidating the processing and compatibility mechanism of Paeoniae Radix Alba and Atractylodis Macrocephalae Rhizoma.
\end{abstract}

\section{Introduction}

Traditional Chinese medicine (TCM) processing is regarded as a pharmaceutical technology based on TCM theory, the requirements of different syndrome treatment, the quality nature of medicine, and different demands of clinical dispensing and preparations [1]. It is one of the characteristics in application of TCM. The compatible components of prescription are composed of prepared Chinese crude drugs after TCM processing.

The prescription compatibility and TCM processing are not only two major features of clinical medication in TCM, but are also critical to distinguish TCM from natural medicine. The research on structural features, compatible effect, and material basis of the herbal pair is the important support in the study of the prescription compatibility since the herbal pair is the minimum unit in prescription of TCM $[2,3]$. They play a guidance and significant role in reveal of the compatibility rule and the scientific connotation. The herbal pair compatibility theory can explain the relationship of the prescription compatibility to some extent. The research on the relationship between the herbal pair compatibility and the prescription compatibility contributes to the elucidation of the prescription compatibility mechanism and the action mechanism of treatment. There are many herbal pairs commonly used in the clinical practice of TCM, such as the herbal pairs of Paeonia Lactiflora-Liquorice, GinsengAconite, and Aconite-Rhizome Zingiberis $[4,5]$ besides the 
Table 1: Major chemical constituents identified in crude and processed Paeoniae Radix Alba and in crude and processed Paeoniae Radix Alba-Atractylodis Macrocephalae Rhizoma herbal pair.

\begin{tabular}{|c|c|c|c|c|c|c|c|}
\hline \multirow[t]{2}{*}{ No. } & \multirow[t]{2}{*}{$t_{R}(\min )$} & \multirow[t]{2}{*}{ Compound name } & \multirow[t]{2}{*}{ Formula } & \multicolumn{2}{|c|}{ Paeoniae Radix Alba } & \multicolumn{2}{|c|}{$\begin{array}{c}\text { Paeoniae Radix } \\
\text { Alba-Atractylodis } \\
\text { Macrocephalae Rhizoma } \\
\text { herbal pair } \\
\text { (Measured area) }\end{array}$} \\
\hline & & & & Crude & Processed & Crude & Processed \\
\hline 1 & 0.84 & 6-O-galloylsucrose & $\mathrm{C}_{19} \mathrm{H}_{26} \mathrm{O}_{15}$ & $1.8570 E+08$ & $1.9012 E+08$ & $4.2870 E+07$ & $4.4158 E+07$ \\
\hline 2 & 0.84 & Glucogallin & $\mathrm{C}_{13} \mathrm{H}_{16} \mathrm{O}_{10}$ & $2.9739 E+08$ & $2.5698 E+08$ & $1.0931 E+08$ & - \\
\hline 3 & 1.05 & Desbenzoylpaeoniflorin & $\mathrm{C}_{16} \mathrm{H}_{24} \mathrm{O}_{10}$ & $1.6682 E+08$ & $1.6263 E+08$ & $9.8500 E+07$ & - \\
\hline 4 & 1.06 & $1^{\prime}$-O-galloylsucrose & $\mathrm{C}_{19} \mathrm{H}_{26} \mathrm{O}_{15}$ & $3.2574 E+08$ & $2.9123 E+08$ & - & - \\
\hline 5 & 1.07 & $\begin{array}{l}\text { 1-O-glucopyranosyl } \\
\text { paeonisuffrone }\end{array}$ & $\mathrm{C}_{16} \mathrm{H}_{24} \mathrm{O}_{9}$ & $2.8667 E+08$ & $2.3654 E+08$ & $1.1532 E+08$ & - \\
\hline 6 & 1.13 & Gallic acid & $\mathrm{C}_{7} \mathrm{H}_{6} \mathrm{O}_{5}$ & $4.1152 E+09$ & $4.0736 E+09$ & $2.7186 E+09$ & $3.1711 E+09$ \\
\hline 7 & 1.18 & Oxypaeoniflorin sulfonate & $\mathrm{C}_{23} \mathrm{H}_{28} \mathrm{O}_{14} \mathrm{~S}$ & $4.9527 E+07$ & $3.5407 E+07$ & $6.1568 E+06$ & $8.5010 E+07$ \\
\hline 8 & 1.22 & Ethyl gallate & $\mathrm{C}_{9} \mathrm{H}_{10} \mathrm{O}_{5}$ & $5.1351 E+07$ & $6.7200 E+07$ & $1.5592 E+07$ & $4.8337 E+07$ \\
\hline 9 & 1.22 & $\begin{array}{l}\text { 6-O-galloyl } \\
\text { desbenzoylpaeoniflorin }\end{array}$ & $\mathrm{C}_{23} \mathrm{H}_{28} \mathrm{O}_{14}$ & $9.9020 E+07$ & $9.5040 E+07$ & $5.3798 E+07$ & - \\
\hline 10 & 1.26 & $\begin{array}{l}\text { 6-O-glucopyranosyl- } \\
\text { lactinolide }\end{array}$ & $\mathrm{C}_{16} \mathrm{H}_{26} \mathrm{O}_{9}$ & $1.0875 E+08$ & $1.1180 E+08$ & - & $3.7130 E+07$ \\
\hline 11 & 1.30 & Paeoniflorin sulfonate I & $\mathrm{C}_{23} \mathrm{H}_{28} \mathrm{O}_{13} \mathrm{~S}$ & $5.3777 E+07$ & $3.6391 E+07$ & $7.3077 E+06$ & $7.7185 E+07$ \\
\hline 12 & 1.30 & Mudanpioside E sulfonate & $\mathrm{C}_{24} \mathrm{H}_{30} \mathrm{O}_{15} \mathrm{~S}$ & $5.3777 E+07$ & $3.6391 E+07$ & $7.3077 E+06$ & $7.7185 E+07$ \\
\hline 13 & 1.43 & $\begin{array}{l}\text { 6-O-glucopyranosyl- } \\
\text { lactinolide }\end{array}$ & $\mathrm{C}_{16} \mathrm{H}_{26} \mathrm{O}_{9}$ & $7.4342 E+08$ & $6.5904 E+08$ & $4.0712 E+08$ & $3.1407 E+08$ \\
\hline 14 & 1.64 & Mudanpioside F & $\mathrm{C}_{16} \mathrm{H}_{24} \mathrm{O}_{8}$ & $6.4178 E+08$ & $6.0980 E+08$ & $4.0130 E+08$ & $6.6680 E+07$ \\
\hline 15 & 1.76 & $\begin{array}{l}\text { Isomaltopaeoniflorin } \\
\text { sulfonate }\end{array}$ & $\mathrm{C}_{29} \mathrm{H}_{38} \mathrm{O}_{18} \mathrm{~S}$ & $1.8858 E+09$ & $1.1382 E+09$ & $2.6277 E+08$ & $5.8622 E+07$ \\
\hline 16 & 1.81 & Pedunculagin & $\mathrm{C}_{34} \mathrm{H}_{24} \mathrm{O}_{22}$ & $4.8098 E+07$ & - & $5.6076 E+07$ & $1.2660 E+09$ \\
\hline 17 & 1.97 & Paeoniflorin sulfonate I & $\mathrm{C}_{23} \mathrm{H}_{28} \mathrm{O}_{13} \mathrm{~S}$ & $3.1881 E+10$ & $2.3387 E+10$ & $6.6202 E+09$ & $5.5241 E+10$ \\
\hline 18 & 2.25 & Oxypaeoniflorin & $\mathrm{C}_{23} \mathrm{H}_{28} \mathrm{O}_{12}$ & $2.3173 E+09$ & $2.4115 E+09$ & $1.6734 E+09$ & $1.4513 E+09$ \\
\hline 19 & 2.36 & Gallotannin & $\mathrm{C}_{27} \mathrm{H}_{24} \mathrm{O}_{18}$ & $2.2850 E+08$ & $2.2458 E+08$ & $1.6284 E+08$ & $1.6703 E+08$ \\
\hline 20 & 2.37 & 1-O-benzoylsucrose & $\mathrm{C}_{19} \mathrm{H}_{26} \mathrm{O}_{12}$ & $1.3761 E+08$ & $1.3161 E+08$ & $1.1673 E+08$ & $8.2986 E+07$ \\
\hline 21 & 2.41 & d-catechin & $\mathrm{C}_{15} \mathrm{H}_{14} \mathrm{O}_{6}$ & $3.7822 E+09$ & $4.2278 E+09$ & $2.6339 E+09$ & $2.5982 E+09$ \\
\hline 22 & 2.63 & Methyl gallate & $\mathrm{C}_{8} \mathrm{H}_{8} \mathrm{O}_{5}$ & $2.3823 E+10$ & $2.4116 E+10$ & $2.3388 E+10$ & - \\
\hline 23 & 2.63 & Salicylic acid & $\mathrm{C}_{7} \mathrm{H}_{6} \mathrm{O}_{3}$ & $2.3823 E+10$ & $2.4116 E+10$ & $2.3388 E+10$ & $1.7399 E+10$ \\
\hline 24 & 2.72 & Albiflorin R1 & $\mathrm{C}_{23} \mathrm{H}_{28} \mathrm{O}_{11}$ & $5.2469 E+08$ & $5.6725 E+08$ & $5.6329 E+08$ & $4.5647 E+08$ \\
\hline 25 & 3.00 & $\begin{array}{l}\text { Kaempferol-3,7-di-O- } \\
\text { glucoside }\end{array}$ & $\mathrm{C}_{27} \mathrm{H}_{30} \mathrm{O}_{16}$ & $3.8065 E+07$ & $2.1896 E+07$ & $3.5719 E+07$ & $1.5513 E+07$ \\
\hline 26 & 3.00 & Paeonoside & $\mathrm{C}_{27} \mathrm{H}_{30} \mathrm{O}_{16}$ & $3.8065 E+07$ & $2.1896 E+07$ & $3.5719 E+07$ & $1.5513 E+07$ \\
\hline 27 & 3.46 & Galloylpaeoniflorin & $\mathrm{C}_{30} \mathrm{H}_{32} \mathrm{O}_{15}$ & $1.3912 E+08$ & $1.5200 E+08$ & $1.1501 E+08$ & $1.0863 E+08$ \\
\hline 28 & 3.47 & Paeonolide & $\mathrm{C}_{20} \mathrm{H}_{28} \mathrm{O}_{12}$ & $1.0622 E+07$ & $1.1936 E+07$ & $9.1812 E+06$ & - \\
\hline 29 & 3.58 & $\begin{array}{l}\text { 6-O-glucopyranosyl- } \\
\text { lactinolide }\end{array}$ & $\mathrm{C}_{16} \mathrm{H}_{26} \mathrm{O}_{9}$ & $2.5249 E+08$ & $2.3834 E+08$ & $2.2675 E+08$ & $2.1241 E+08$ \\
\hline 30 & 3.68 & Oxypaeoniflorin & $\mathrm{C}_{23} \mathrm{H}_{28} \mathrm{O}_{12}$ & $1.5407 E+08$ & - & $1.4345 E+08$ & $1.3307 E+08$ \\
\hline 31 & 3.76 & $\begin{array}{l}\text { 6-O-glucopyranosyl- } \\
\text { lactinolide }\end{array}$ & $\mathrm{C}_{16} \mathrm{H}_{26} \mathrm{O}_{9}$ & $3.2664 E+08$ & - & $3.0588 E+08$ & $3.1627 E+08$ \\
\hline 32 & 3.88 & Paeonilactone B & $\mathrm{C}_{10} \mathrm{H}_{12} \mathrm{O}_{4}$ & $9.0325 E+07$ & $9.3539 E+07$ & $5.1257 E+07$ & $8.9597 E+07$ \\
\hline 33 & 3.93 & Isomaltopaeoniflorin & $\mathrm{C}_{29} \mathrm{H}_{38} \mathrm{O}_{16}$ & $1.1545 E+10$ & $1.1941 E+10$ & $1.2282 E+10$ & $9.4600 E+09$ \\
\hline 34 & 4.07 & Albiflorin & $\mathrm{C}_{23} \mathrm{H}_{28} \mathrm{O}_{11}$ & $2.9587 E+10$ & $2.9296 E+10$ & $2.8430 E+10$ & $2.8684 E+10$ \\
\hline 35 & 4.32 & Glucopyranosylalbiorin & $\mathrm{C}_{29} \mathrm{H}_{38} \mathrm{O}_{16}$ & $2.2813 E+09$ & $2.4109 E+08$ & $2.0383 E+08$ & $1.6844 E+08$ \\
\hline 36 & 4.34 & $\begin{array}{l}\text { Galloylpaeoniflorin } \\
\text { sulfonate }\end{array}$ & $\mathrm{C}_{30} \mathrm{H}_{32} \mathrm{O}_{17} \mathrm{~S}$ & $7.6943 E+08$ & $5.6793 E+08$ & $1.5501 E+08$ & $1.4886 E+09$ \\
\hline
\end{tabular}


TABLE 1: Continued.

\begin{tabular}{|c|c|c|c|c|c|c|c|}
\hline \multirow[t]{2}{*}{ No. } & \multirow[t]{2}{*}{$t_{R}(\min )$} & \multirow[t]{2}{*}{ Compound name } & \multirow[t]{2}{*}{ Formula } & \multicolumn{2}{|c|}{ Paeoniae Radix Alba } & \multicolumn{2}{|c|}{$\begin{array}{c}\text { Paeoniae Radix } \\
\text { Alba-Atractylodis } \\
\text { Macrocephalae Rhizoma } \\
\text { herbal pair } \\
\text { (Measured area) }\end{array}$} \\
\hline & & & & Crude & Processed & Crude & Processed \\
\hline 37 & 4.34 & Galloylpaeoniflorin isomer & $\mathrm{C}_{30} \mathrm{H}_{32} \mathrm{O}_{15}$ & $6.7592 E+08$ & $7.4322 E+08$ & $6.0470 E+08$ & $5.4051 E+08$ \\
\hline 38 & 4.38 & $\begin{array}{l}\text { 1,2,3,6-tetra-O- } \\
\text { galloylglucose }\end{array}$ & $\mathrm{C}_{34} \mathrm{H}_{28} \mathrm{O}_{22}$ & $4.6602 E+08$ & $3.4977 E+08$ & $4.0697 E+08$ & $3.6901 E+08$ \\
\hline 39 & 4.38 & Tetragalloyl glucose A & $\mathrm{C}_{34} \mathrm{H}_{28} \mathrm{O}_{22}$ & $4.6602 E+08$ & $3.4977 E+08$ & $4.0697 E+08$ & $3.6901 E+08$ \\
\hline 40 & 4.56 & Mudanpioside F & $\mathrm{C}_{16} \mathrm{H}_{24} \mathrm{O}_{8}$ & $8.4156 E+07$ & $8.2734 E+07$ & $7.2666 E+07$ & $7.9227 E+07$ \\
\hline 41 & 4.60 & Oxypaeoniflorin isomer & $\mathrm{C}_{23} \mathrm{H}_{28} \mathrm{O}_{12}$ & $9.6610 E+08$ & $9.8706 E+08$ & $9.2464 E+08$ & $8.7359 E+08$ \\
\hline 42 & 4.65 & Gallotannin & $\mathrm{C}_{27} \mathrm{H}_{24} \mathrm{O}_{18}$ & $6.7737 E+07$ & - & - & - \\
\hline 43 & 4.77 & Paeoniflorin & $\mathrm{C}_{23} \mathrm{H}_{28} \mathrm{O}_{11}$ & $5.9556 E+10$ & $6.1356 E+10$ & $5.9929 E+10$ & $5.8832 E+10$ \\
\hline 44 & 4.89 & Paeoniflorin sulfonate II & $\mathrm{C}_{23} \mathrm{H}_{28} \mathrm{O}_{13} \mathrm{~S}$ & $1.1095 E+08$ & $1.4567 E+08$ & $5.5052 E+07$ & $2.7813 E+08$ \\
\hline 45 & 4.98 & $\begin{array}{l}\text { Isogalloylpaeoniflorin } \\
\text { sulfonate }\end{array}$ & $\mathrm{C}_{30} \mathrm{H}_{32} \mathrm{O}_{17} \mathrm{~S}$ & $3.6742 E+07$ & - & - & - \\
\hline 46 & 5.05 & Ethyl gallate & $\mathrm{C}_{9} \mathrm{H}_{10} \mathrm{O}_{5}$ & $6.0669 E+07$ & $5.4850 E+07$ & $1.6681 E+07$ & $2.6790 E+07$ \\
\hline 47 & 5.05 & Methyl salicylate & $\mathrm{C}_{8} \mathrm{H}_{8} \mathrm{O}_{3}$ & $6.0669 E+07$ & $5.4850 E+07$ & $1.6681 E+07$ & $2.6790 E+07$ \\
\hline 48 & 5.15 & Benzoic acid & $\mathrm{C}_{7} \mathrm{H}_{6} \mathrm{O}_{2}$ & $4.0163 E+07$ & $4.5493 E+07$ & $2.9695 E+07$ & $2.9727 E+07$ \\
\hline 49 & 5.25 & Paeonol & $\mathrm{C}_{9} \mathrm{H}_{10} \mathrm{O}_{3}$ & $6.8567 E+07$ & $7.4129 E+07$ & $9.9992 E+07$ & $6.5619 E+07$ \\
\hline 50 & 5.25 & $\begin{array}{l}\text { 4-hydroxy-3-methoxy } \\
\text { acetophenone }\end{array}$ & $\mathrm{C}_{9} \mathrm{H}_{10} \mathrm{O}_{3}$ & $6.8567 E+07$ & $7.4129 E+07$ & $9.9992 E+07$ & $6.5619 E+07$ \\
\hline 51 & 5.31 & ortho-oxypaeoniflorin & $\mathrm{C}_{23} \mathrm{H}_{28} \mathrm{O}_{12}$ & $1.9080 E+09$ & $1.9263 E+09$ & $1.8723 E+09$ & $1.6842 E+09$ \\
\hline 52 & 5.63 & Ethyl gallate & $\mathrm{C}_{9} \mathrm{H}_{10} \mathrm{O}_{5}$ & $1.4627 E+08$ & $1.2812 E+08$ & $1.0365 E+08$ & $8.8155 E+07$ \\
\hline 53 & 5.63 & Methyl salicylate & $\mathrm{C}_{8} \mathrm{H}_{8} \mathrm{O}_{3}$ & $1.4627 E+08$ & $1.2812 E+08$ & $1.0365 E+08$ & $8.8155 E+07$ \\
\hline 54 & 5.66 & Kaempferol-3-O-glucoside & $\mathrm{C}_{21} \mathrm{H}_{20} \mathrm{O}_{11}$ & $1.6012 E+07$ & $1.7385 E+07$ & - & - \\
\hline 55 & 5.66 & Astragalin & $\mathrm{C}_{21} \mathrm{H}_{20} \mathrm{O}_{11}$ & $1.6012 E+07$ & $1.7385 E+07$ & - & - \\
\hline 56 & 6.01 & Eugeniin & $\mathrm{C}_{41} \mathrm{H}_{30} \mathrm{O}_{26}$ & $2.7483 E+08$ & $3.0279 E+08$ & $2.8080 E+08$ & $3.0479 E+08$ \\
\hline 57 & 6.01 & $\begin{array}{l}\text { Dihydroxymethyl benzoyl } \\
\text { tetragalloyl glucose }\end{array}$ & $\mathrm{C}_{41} \mathrm{H}_{30} \mathrm{O}_{26}$ & $2.7483 E+08$ & $3.0279 E+08$ & $2.8080 E+08$ & $3.0479 E+08$ \\
\hline 58 & 6.03 & $\begin{array}{l}\text { 1,2,3,6-tetra-O- } \\
\text { galloylglucose isomer } \\
\text { A }\end{array}$ & $\mathrm{C}_{34} \mathrm{H}_{28} \mathrm{O}_{22}$ & $1.3555 E+09$ & - & $1.1980 E+09$ & $1.1039 E+09$ \\
\hline 59 & 6.03 & Tetragalloyl glucose B & $\mathrm{C}_{34} \mathrm{H}_{28} \mathrm{O}_{22}$ & $1.3555 E+09$ & - & - & - \\
\hline 60 & 6.08 & Astragalin & $\mathrm{C}_{21} \mathrm{H}_{20} \mathrm{O}_{11}$ & $1.5009 E+07$ & $1.8552 E+07$ & $1.5922 E+07$ & $1.4002 E+07$ \\
\hline 61 & 6.09 & $\begin{array}{l}\text { Isomaltopaeoniflorin } \\
\text { isomer }\end{array}$ & $\mathrm{C}_{29} \mathrm{H}_{38} \mathrm{O}_{16}$ & $7.5172 E+07$ & - & - & - \\
\hline 62 & 6.47 & $\begin{array}{l}\text { 1,2,3,6-tetra-O- } \\
\text { galloylglucose isomer } \\
\mathrm{B}\end{array}$ & $\mathrm{C}_{34} \mathrm{H}_{28} \mathrm{O}_{22}$ & $1.5882 E+09$ & - & - & $1.2570 E+09$ \\
\hline 63 & 6.47 & Tetragalloyl glucose C & $\mathrm{C}_{34} \mathrm{H}_{28} \mathrm{O}_{22}$ & $1.5882 E+09$ & - & - & $1.2570 E+09$ \\
\hline 64 & 6.85 & 3,6-di-O-galloyl paeoniorin & $\mathrm{C}_{37} \mathrm{H}_{36} \mathrm{O}_{19}$ & $7.6512 E+07$ & - & - & - \\
\hline 65 & 6.96 & $\begin{array}{l}\text { 1,2,3,6-tetra-O- } \\
\text { galloylglucose }\end{array}$ & $\mathrm{C}_{34} \mathrm{H}_{28} \mathrm{O}_{22}$ & $4.4729 E+08$ & $4.5825 E+08$ & $4.0642 E+08$ & $4.2393 E+08$ \\
\hline 66 & 6.96 & Tetragalloyl glucose D & $\mathrm{C}_{34} \mathrm{H}_{28} \mathrm{O}_{22}$ & $4.4729 E+08$ & $4.5825 E+08$ & $4.0642 E+08$ & $4.2393 E+08$ \\
\hline 67 & 7.35 & $\begin{array}{l}\text { Galloylpaeoniflorin isomer } \\
\text { I }\end{array}$ & $\mathrm{C}_{30} \mathrm{H}_{32} \mathrm{O}_{15}$ & $1.2156 E+10$ & $1.2451 E+10$ & $1.1484 E+10$ & $1.0962 E+10$ \\
\hline 68 & 7.60 & $\begin{array}{l}\text { 1-O-glucopyranosyl-8-O- } \\
\text { benzoyl } \\
\text { paeonisuffrone }\end{array}$ & $\mathrm{C}_{23} \mathrm{H}_{28} \mathrm{O}_{10}$ & $4.3983 E+07$ & $4.5347 E+07$ & $4.4927 E+07$ & $3.9869 E+07$ \\
\hline 69 & 7.71 & $\begin{array}{l}\text { Glucopyranosylalbiorin } \\
\text { isomer I }\end{array}$ & $\mathrm{C}_{29} \mathrm{H}_{38} \mathrm{O}_{16}$ & $7.2982 E+07$ & $7.9341 E+07$ & - & $1.8872 E+07$ \\
\hline
\end{tabular}


TABle 1: Continued.

\begin{tabular}{|c|c|c|c|c|c|c|c|}
\hline \multirow[t]{2}{*}{ No. } & \multirow[t]{2}{*}{$t_{R}(\min )$} & \multirow[t]{2}{*}{ Compound name } & \multirow[t]{2}{*}{ Formula } & \multicolumn{2}{|c|}{ Paeoniae Radix Alba } & \multicolumn{2}{|c|}{$\begin{array}{c}\text { Paeoniae Radix } \\
\text { Alba-Atractylodis } \\
\text { Macrocephalae Rhizoma } \\
\text { herbal pair } \\
\text { (Measured area) }\end{array}$} \\
\hline & & & & Crude & Processed & Crude & Processed \\
\hline 70 & 8.18 & $\begin{array}{l}\text { 1-O-glucopyranosyl-8-O- } \\
\text { benzoyl } \\
\text { paeonisuffrone }\end{array}$ & $\mathrm{C}_{23} \mathrm{H}_{28} \mathrm{O}_{10}$ & $7.4648 E+07$ & $8.3204 E+07$ & $6.5957 E+07$ & $5.9832 E+07$ \\
\hline 71 & 8.31 & Ortho-oxypaeoniflorin & $\mathrm{C}_{23} \mathrm{H}_{28} \mathrm{O}_{12}$ & $2.4469 E+07$ & $2.4504 E+07$ & $2.3932 E+07$ & $2.2796 E+07$ \\
\hline 72 & 8.45 & $\begin{array}{l}\text { 1,2,3,4,6-Penta-O-galloyl- } \\
\text { D-glucopyranose }\end{array}$ & $\mathrm{C}_{41} \mathrm{H}_{32} \mathrm{O}_{26}$ & $1.1843 E+10$ & $1.0905 E+10$ & $1.0518 E+10$ & $1.0489 E+10$ \\
\hline 73 & 8.45 & Pentagalloyl glucose & $\mathrm{C}_{41} \mathrm{H}_{32} \mathrm{O}_{26}$ & $1.1843 E+10$ & $1.0905 E+10$ & $1.0518 E+10$ & $1.0489 E+10$ \\
\hline 74 & 8.64 & Lactiflorin & $\mathrm{C}_{23} \mathrm{H}_{26} \mathrm{O}_{10}$ & $1.0818 E+08$ & $1.8628 E+08$ & $1.3689 E+08$ & - \\
\hline 75 & 8.80 & Galloylalbiroin & $\mathrm{C}_{30} \mathrm{H}_{32} \mathrm{O}_{15}$ & $3.2696 E+09$ & - & - & - \\
\hline 76 & 9.17 & Astragalin & $\mathrm{C}_{21} \mathrm{H}_{20} \mathrm{O}_{11}$ & $1.0717 E+07$ & $1.3960 E+07$ & $1.2843 E+07$ & $1.0582 E+07$ \\
\hline 77 & 9.25 & Lactinolide & $\mathrm{C}_{10} \mathrm{H}_{16} \mathrm{O}_{4}$ & $2.7251 E+07$ & $2.6105 E+07$ & $2.1735 E+07$ & $3.2770 E+07$ \\
\hline 78 & 9.29 & $\begin{array}{l}\text { Galloylpaeoniflorin isomer } \\
\text { II }\end{array}$ & $\mathrm{C}_{30} \mathrm{H}_{32} \mathrm{O}_{15}$ & $2.8831 E+09$ & - & $2.6829 E+09$ & $2.2850 E+09$ \\
\hline 79 & 9.68 & $\begin{array}{l}\text { Glucopyranosylalbiorin } \\
\text { isomer II }\end{array}$ & $\mathrm{C}_{29} \mathrm{H}_{38} \mathrm{O}_{16}$ & $2.4321 E+07$ & $2.6804 E+07$ & $2.2576 E+07$ & $2.4950 E+07$ \\
\hline 80 & 9.84 & Hexagalloyl glucose & $\mathrm{C}_{48} \mathrm{H}_{36} \mathrm{O}_{30}$ & $4.9153 E+07$ & - & $6.8676 E+08$ & $5.7793 E+08$ \\
\hline 81 & 9.95 & $\begin{array}{l}\text { Oxybenzoyl- } \\
\text { oxypaeoniflorin }\end{array}$ & $\mathrm{C}_{30} \mathrm{H}_{32} \mathrm{O}_{14}$ & $1.4385 E+07$ & $1.6654 E+07$ & $1.1051 E+07$ & $1.1345 E+07$ \\
\hline 82 & 10.07 & $\begin{array}{l}\text { 1-O-glucopyranosyl-8-O- } \\
\text { benzoylpaeonisuffrone }\end{array}$ & $\mathrm{C}_{23} \mathrm{H}_{28} \mathrm{O}_{10}$ & $3.6916 E+09$ & $3.5634 E+09$ & $3.1333 E+09$ & $3.2106 E+09$ \\
\hline 83 & 10.29 & Albiflorin $\mathrm{R} 1$ isomer I & $\mathrm{C}_{23} \mathrm{H}_{28} \mathrm{O}_{11}$ & $6.3346 E+09$ & $6.6205 E+09$ & $5.9528 E+09$ & $5.8736 E+09$ \\
\hline 84 & 10.74 & Hexagalloyl glucose & $\mathrm{C}_{48} \mathrm{H}_{36} \mathrm{O}_{30}$ & $4.9225 E+08$ & $2.5582 E+08$ & $1.9395 E+09$ & $1.5439 E+09$ \\
\hline 85 & 10.76 & Lactiflorin & $\mathrm{C}_{23} \mathrm{H}_{26} \mathrm{O}_{10}$ & $1.2785 E+09$ & $3.5174 E+09$ & $9.9713 E+08$ & $3.4524 E+09$ \\
\hline 86 & 10.84 & $\begin{array}{l}\text { Benzoylpaeoniflorin } \\
\text { Sulfonate }\end{array}$ & $\mathrm{C}_{30} \mathrm{H}_{32} \mathrm{O}_{14} \mathrm{~S}$ & $9.0616 E+08$ & $6.4075 E+08$ & $1.5946 E+08$ & $2.1931 E+09$ \\
\hline 87 & 10.88 & 3,6-di-O-galloyl paeoniorin & $\mathrm{C}_{37} \mathrm{H}_{36} \mathrm{O}_{19}$ & $1.6123 E+08$ & - & - & - \\
\hline 88 & 10.95 & $\begin{array}{l}\text { Ortho-oxypaeoniflorin } \\
\text { isomer }\end{array}$ & $\mathrm{C}_{23} \mathrm{H}_{28} \mathrm{O}_{12}$ & $5.5563 E+07$ & $5.8774 E+07$ & $5.7147 E+07$ & $5.6640 E+07$ \\
\hline 89 & 11.52 & 3,6-di-O-galloyl paeoniorin & $\mathrm{C}_{37} \mathrm{H}_{36} \mathrm{O}_{19}$ & $3.6509 E+08$ & $3.9290 E+08$ & $5.2162 E+08$ & $5.3781 E+08$ \\
\hline 90 & 11.72 & $\begin{array}{l}\text { 3,6-di-O-galloyl paeoniorin } \\
\text { isomer }\end{array}$ & $\mathrm{C}_{37} \mathrm{H}_{36} \mathrm{O}_{19}$ & $9.7356 E+08$ & - & $1.2523 E+09$ & $9.5929 E+08$ \\
\hline 91 & 11.75 & Galloylalbiroin isomer I & $\mathrm{C}_{30} \mathrm{H}_{32} \mathrm{O}_{15}$ & $2.3457 E+08$ & - & - & - \\
\hline 92 & 11.84 & $\begin{array}{l}\text { Oxypaeoniflorin sulfonate } \\
\text { isomer }\end{array}$ & $\mathrm{C}_{23} \mathrm{H}_{28} \mathrm{O}_{14} \mathrm{~S}$ & $2.1063 E+07$ & $1.9747 E+07$ & $1.3875 E+07$ & $1.0840 E+07$ \\
\hline 93 & 12.15 & $\begin{array}{l}\text { 1-O-glucopyranosyl-8-O- } \\
\text { benzoylpaeonisuffrone }\end{array}$ & $\mathrm{C}_{23} \mathrm{H}_{28} \mathrm{O}_{10}$ & $7.2104 E+07$ & $7.1468 E+07$ & $6.7309 E+07$ & $6.5917 E+07$ \\
\hline 94 & 12.15 & $\begin{array}{l}\text { Oxybenzoyl- } \\
\text { oxypaeoniflorin }\end{array}$ & $\mathrm{C}_{30} \mathrm{H}_{32} \mathrm{O}_{14}$ & $1.9982 E+08$ & - & - & $1.6891 E+08$ \\
\hline 95 & 12.18 & Benzoyloxypaeoniflorin & $\mathrm{C}_{30} \mathrm{H}_{32} \mathrm{O}_{13}$ & $2.0822 E+08$ & - & $2.0163 E+08$ & $1.9074 E+08$ \\
\hline 96 & 13.42 & $\begin{array}{l}\text { Benzoyloxypaeoniflorin } \\
\text { isomer }\end{array}$ & $\mathrm{C}_{30} \mathrm{H}_{32} \mathrm{O}_{13}$ & $8.6458 E+07$ & $6.2282 E+07$ & $7.6048 E+07$ & $7.2791 E+07$ \\
\hline 97 & 13.44 & $\begin{array}{l}\text { Oxybenzoyl- } \\
\text { oxypaeoniflorin isomer } \\
\text { I }\end{array}$ & $\mathrm{C}_{30} \mathrm{H}_{32} \mathrm{O}_{14}$ & $1.4728 E+07$ & $1.7389 E+07$ & $1.5360 E+07$ & $1.6008 E+07$ \\
\hline 98 & 13.85 & Galloylalbiroin isomer II & $\mathrm{C}_{30} \mathrm{H}_{32} \mathrm{O}_{15}$ & $9.6403 E+07$ & $1.2196 E+08$ & $1.0506 E+08$ & $1.0272 E+08$ \\
\hline 99 & 14.05 & $\begin{array}{l}\text { Oxybenzoyl- } \\
\text { oxypaeoniflorin isomer } \\
\text { II }\end{array}$ & $\mathrm{C}_{30} \mathrm{H}_{32} \mathrm{O}_{14}$ & $2.5323 E+07$ & $2.9603 E+07$ & $2.3556 E+07$ & $2.8526 E+07$ \\
\hline 100 & 14.13 & Benzoyloxypaeoniflorin & $\mathrm{C}_{30} \mathrm{H}_{32} \mathrm{O}_{13}$ & $3.8096 E+07$ & $3.8557 E+07$ & $3.7499 E+07$ & $3.5800 E+07$ \\
\hline
\end{tabular}


TABLe 1: Continued.

\begin{tabular}{|c|c|c|c|c|c|c|c|}
\hline \multirow[t]{2}{*}{ No. } & \multirow[t]{2}{*}{$t_{R}(\min )$} & \multirow[t]{2}{*}{ Compound name } & \multirow[t]{2}{*}{ Formula } & \multicolumn{2}{|c|}{ Paeoniae Radix Alba } & \multicolumn{2}{|c|}{$\begin{array}{c}\text { Paeoniae Radix } \\
\text { Alba-Atractylodis } \\
\text { Macrocephalae Rhizoma } \\
\text { herbal pair } \\
\text { (Measured area) }\end{array}$} \\
\hline & & & & Crude & Processed & Crude & Processed \\
\hline 101 & 15.07 & $\begin{array}{l}\text { Benzoyloxypaeoniflorin } \\
\text { isomer I }\end{array}$ & $\mathrm{C}_{30} \mathrm{H}_{32} \mathrm{O}_{13}$ & $1.9827 E+07$ & $2.3616 E+07$ & - & - \\
\hline 102 & 15.38 & $\begin{array}{l}\text { Benzoyloxypaeoniflorin } \\
\text { isomer II }\end{array}$ & $\mathrm{C}_{30} \mathrm{H}_{32} \mathrm{O}_{13}$ & $1.1841 E+07$ & $1.3730 E+07$ & - & - \\
\hline 103 & 16.01 & Oxybenzoyl-paeoniflorin & $\mathrm{C}_{30} \mathrm{H}_{32} \mathrm{O}_{12}$ & $1.8152 E+07$ & - & $1.8435 E+07$ & - \\
\hline 104 & 16.95 & Isobenzoylpaeoniflorin & $\mathrm{C}_{30} \mathrm{H}_{32} \mathrm{O}_{12}$ & $1.2225 E+10$ & $1.3228 E+10$ & $1.2158 E+10$ & $1.2391 E+10$ \\
\hline 105 & 16.95 & $\begin{array}{l}\text { Oxybenzoyl-paeoniflorin } \\
\text { isomer I }\end{array}$ & $\mathrm{C}_{30} \mathrm{H}_{32} \mathrm{O}_{12}$ & $1.2225 E+10$ & $1.3228 E+10$ & $1.2158 E+10$ & $1.2391 E+10$ \\
\hline 106 & 17.23 & $\begin{array}{l}\text { Benzoylpaeoniflorin } \\
\text { Sulfonate }\end{array}$ & $\mathrm{C}_{30} \mathrm{H}_{32} \mathrm{O}_{14} \mathrm{~S}$ & $1.5680 E+07$ & $1.2235 E+07$ & $5.6573 E+06$ & $3.5831 E+07$ \\
\hline 107 & 17.48 & $\begin{array}{l}\text { Isobenzoylpaeoniflorin } \\
\text { isomer I }\end{array}$ & $\mathrm{C}_{30} \mathrm{H}_{32} \mathrm{O}_{12}$ & $5.4138 E+09$ & $5.4432 E+09$ & $5.2522 E+09$ & $5.3238 E+09$ \\
\hline 108 & 17.48 & $\begin{array}{l}\text { Oxybenzoyl-paeoniflorin } \\
\text { isomer II }\end{array}$ & $\mathrm{C}_{30} \mathrm{H}_{32} \mathrm{O}_{12}$ & $5.4138 E+09$ & $5.4432 E+09$ & $5.2522 E+09$ & $5.3238 E+09$ \\
\hline 109 & 17.86 & Benzoyloxypaeoniflorin & $\mathrm{C}_{30} \mathrm{H}_{32} \mathrm{O}_{13}$ & $3.4347 E+07$ & $3.4852 E+07$ & $3.5980 E+07$ & $3.8814 E+07$ \\
\hline 110 & 18.55 & $\begin{array}{l}\text { Benzoyloxypaeoniflorin } \\
\text { isomer }\end{array}$ & $\mathrm{C}_{30} \mathrm{H}_{32} \mathrm{O}_{13}$ & $1.5397 E+07$ & $1.7656 E+07$ & $1.7246 E+07$ & $1.8012 E+07$ \\
\hline 111 & 18.69 & Albiflorin R1 isomer II & $\mathrm{C}_{23} \mathrm{H}_{28} \mathrm{O}_{11}$ & $2.0046 E+07$ & $1.9851 E+07$ & $2.3462 E+07$ & - \\
\hline 112 & 19.30 & Albiflorin R1 isomer III & $\mathrm{C}_{23} \mathrm{H}_{28} \mathrm{O}_{11}$ & $2.9827 E+06$ & - & - & $5.6105 E+06$ \\
\hline 113 & 21.79 & Palbinone & $\mathrm{C}_{22} \mathrm{H}_{30} \mathrm{O}_{4}$ & $8.9687 E+07$ & $1.3174 E+08$ & $1.2834 E+08$ & $5.7610 E+07$ \\
\hline 114 & 21.93 & $\begin{array}{l}\text { Isobenzoylpaeoniflorin } \\
\text { isomer II }\end{array}$ & $\mathrm{C}_{30} \mathrm{H}_{32} \mathrm{O}_{12}$ & $4.5356 E+08$ & $4.2874 E+07$ & $3.4016 E+08$ & $2.7347 E+08$ \\
\hline 115 & 21.93 & $\begin{array}{l}\text { Oxybenzoyl-paeoniflorin } \\
\text { isomer III }\end{array}$ & $\mathrm{C}_{30} \mathrm{H}_{32} \mathrm{O}_{12}$ & $4.5356 E+08$ & $4.2874 E+07$ & $3.4016 E+08$ & $2.7347 E+08$ \\
\hline 116 & 22.15 & Paeonilactinone & $\mathrm{C}_{10} \mathrm{H}_{16} \mathrm{O}_{2}$ & $7.0423 E+06$ & $3.7108 E+06$ & $8.0036 E+06$ & $6.6886 E+06$ \\
\hline 117 & 36.46 & Hederagenin & $\mathrm{C}_{30} \mathrm{H}_{48} \mathrm{O}_{4}$ & $7.6725 E+07$ & $8.1456 E+07$ & $9.7498 E+07$ & $4.7332 E+07$ \\
\hline 118 & 37.31 & 23-hydroxybetulinic acid & $\mathrm{C}_{30} \mathrm{H}_{48} \mathrm{O}_{4}$ & $3.9836 E+07$ & $4.0995 E+07$ & $3.9906 E+07$ & $2.2611 E+07$ \\
\hline 119 & 38.14 & Astrantiagenin D & $\mathrm{C}_{30} \mathrm{H}_{46} \mathrm{O}_{4}$ & $7.8714 E+06$ & $7.9560 E+06$ & $1.1904 E+07$ & $3.8958 E+06$ \\
\hline 120 & 43.00 & Astrantiagenin D isomer & $\mathrm{C}_{30} \mathrm{H}_{46} \mathrm{O}_{4}$ & $4.0450 E+06$ & - & $3.1585 E+06$ & - \\
\hline 121 & 45.65 & Oleanolic acid & $\mathrm{C}_{30} \mathrm{H}_{48} \mathrm{O}_{3}$ & $1.1266 E+08$ & $9.4258 E+07$ & $7.6434 E+07$ & $4.3295 E+07$ \\
\hline 122 & 46.10 & Betulinic acid & $\mathrm{C}_{30} \mathrm{H}_{48} \mathrm{O}_{3}$ & $6.2494 E+06$ & $2.3289 E+07$ & $4.0543 E+07$ & $2.3912 E+07$ \\
\hline 123 & 52.48 & Daucosterol & $\mathrm{C}_{35} \mathrm{H}_{60} \mathrm{O}_{6}$ & $1.4060 E+07$ & $1.9624 E+07$ & $8.5440 E+06$ & $6.3156 E+06$ \\
\hline
\end{tabular}

Paeoniae Radix Alba-Atractylodis Macrocephalae Rhizoma herbal pair frequently used in all China dynasties [6, 7]. Paeoniae Radix Alba nourishes blood and liver, and Atractylodis Macrocephalae Rhizoma helps invigorate spleen and eliminate dampness [8-12]. Thus, the compatibility of these two medicines could help achieve the goal of purging wood from the earth, regulating the functions of liver and spleen, benefiting qi, and nourishing blood [13-15]. Although the compositions of these two medicines have been extensively studied, the appropriate processing method of them, such as frying, which is believed by the practitioners of traditional medicine to have the effects for enhancing the efficacy of the medicine, and their underlying compatibility mechanism are still under investigation.
The objective of this study is to investigate the qualitative, preprocessing, and postprocessing changes in the composition and compatibility of Paeoniae Radix Alba and Atractylodis Macrocephalae Rhizoma by using Q Exactive hybrid quadrupole-Orbitrap mass spectrometer combined with high-performance quadrupole precursor selection with high-resolution and accurate-mass Orbitrap detection. The work could serve as a theoretical basis for the development of medicines from Paeoniae Radix Alba and Atractylodis Macrocephalae Rhizoma, and the reasonable clinical medication. Furthermore, it provides new insights into the investigation of the herbal pair and for the study of the appropriate processing method for Chinese herbal medicines and their underlying compatibility mechanism. 
TABLE 2: Major chemical constituents identified in crude and processed Atractylodis Macrocephalae Rhizoma and in crude and processed Paeoniae Radix Alba-Atractylodis Macrocephalae Rhizoma herbal pair.

\begin{tabular}{|c|c|c|c|c|c|c|c|}
\hline \multirow[t]{2}{*}{ No. } & \multirow[t]{2}{*}{$t_{R}(\min )$} & \multirow[t]{2}{*}{ Compound name } & \multirow[t]{2}{*}{ Formula } & \multicolumn{2}{|c|}{ Atractylodis Macrocephalae Rhizoma } & \multicolumn{2}{|c|}{$\begin{array}{c}\text { Paeoniae Radix } \\
\text { Alba-Atractylodis } \\
\text { Macrocephalae Rhizoma } \\
\text { herbal pair } \\
\text { (Measured area) }\end{array}$} \\
\hline & & & & Crude & Processed & Crude & Processed \\
\hline 1 & 1.72 & Protocatechuic acid & $\mathrm{C}_{7} \mathrm{H}_{6} \mathrm{O}_{4}$ & $2.0389 E+07$ & $1.4454 E+07$ & $2.0881 E+07$ & $2.4383 E+07$ \\
\hline 2 & 2.67 & $\begin{array}{l}\text { Protocatechuic acid isomer } \\
\text { I }\end{array}$ & $\mathrm{C}_{7} \mathrm{H}_{6} \mathrm{O}_{4}$ & $9.6661 E+07$ & - & - & - \\
\hline 3 & 3.24 & Caffeic acid & $\mathrm{C}_{9} \mathrm{H}_{8} \mathrm{O}_{4}$ & $3.6818 E+08$ & $1.7393 E+08$ & $2.8796 E+08$ & $1.2882 E+08$ \\
\hline 4 & 3.73 & $\begin{array}{l}\text { Protocatechuic acid isomer } \\
\text { II }\end{array}$ & $\mathrm{C}_{7} \mathrm{H}_{6} \mathrm{O}_{4}$ & $2.0846 E+07$ & - & - & $1.2022 E+07$ \\
\hline 5 & 4.21 & Dictamnoside A isomer I & $\mathrm{C}_{21} \mathrm{H}_{36} \mathrm{O}_{9}$ & $1.8843 E+07$ & $2.4981 E+07$ & $1.0636 E+07$ & $1.3140 E+07$ \\
\hline 6 & 4.70 & Dictamnoside A isomer II & $\mathrm{C}_{21} \mathrm{H}_{36} \mathrm{O}_{9}$ & $2.8770 E+07$ & $3.4768 E+07$ & $1.0395 E+07$ & $1.4208 E+07$ \\
\hline 7 & 5.63 & Scopoletin & $\mathrm{C}_{10} \mathrm{H}_{8} \mathrm{O}_{4}$ & $6.1458 E+07$ & $4.1494 E+07$ & $6.1562 E+07$ & $5.3342 E+07$ \\
\hline 8 & 5.82 & Dictamnoside A & $\mathrm{C}_{21} \mathrm{H}_{36} \mathrm{O}_{9}$ & $9.6195 E+07$ & $1.1991 E+08$ & $7.5446 E+07$ & $9.4190 E+07$ \\
\hline 9 & 8.77 & Atracetylentriol & $\mathrm{C}_{14} \mathrm{H}_{16} \mathrm{O}_{3}$ & $1.2538 E+07$ & $5.4052 E+06$ & - & - \\
\hline 10 & 9.33 & Ferulic acid & $\mathrm{C}_{10} \mathrm{H}_{10} \mathrm{O}_{4}$ & $1.3958 E+07$ & $9.1214 E+06$ & $1.1912 E+07$ & $9.6849 E+06$ \\
\hline 11 & 25.81 & Atractylenolide I isomer & $\mathrm{C}_{15} \mathrm{H}_{18} \mathrm{O}_{2}$ & $4.5224 E+09$ & $4.2401 E+09$ & $5.9401 E+09$ & $6.5277 E+09$ \\
\hline 12 & 25.83 & Atractylenolide III & $\mathrm{C}_{15} \mathrm{H}_{20} \mathrm{O}_{3}$ & $2.5549 E+09$ & $1.8023 E+09$ & $2.8280 E+09$ & $3.1632 E+09$ \\
\hline 13 & 26.17 & $\begin{array}{l}\text { 12-methylbutyryl-14-acetyl- } \\
2 \mathrm{E}, 8 \mathrm{EZ}, 10 \mathrm{E} \text {-atractylentriol }\end{array}$ & $\mathrm{C}_{21} \mathrm{H}_{26} \mathrm{O}_{5}$ & $2.4755 E+07$ & - & - & - \\
\hline 14 & 26.95 & $\begin{array}{l}\text { 12-methylbutyryl-14-acetyl- } \\
2 \mathrm{E}, 8 \mathrm{EZ}, 10 \mathrm{E} \text {-atractylentriol } \\
\text { isomer }\end{array}$ & $\mathrm{C}_{21} \mathrm{H}_{26} \mathrm{O}_{5}$ & $7.5991 E+07$ & - & - & - \\
\hline 15 & 31.10 & Atractylenolide II isomer & $\mathrm{C}_{15} \mathrm{H}_{20} \mathrm{O}_{2}$ & $6.7883 E+09$ & $4.5794 E+09$ & $7.6246 E+09$ & $7.8814 E+09$ \\
\hline 16 & 31.66 & Atractylenolide II & $\mathrm{C}_{15} \mathrm{H}_{20} \mathrm{O}_{2}$ & $2.8279 E+10$ & $1.9902 E+10$ & $3.0285 E+10$ & $3.1294 E+10$ \\
\hline 17 & 33.44 & Atractylodin & $\mathrm{C}_{13} \mathrm{H}_{10} \mathrm{O}$ & $6.4157 E+06$ & - & $7.0452 E+07$ & - \\
\hline 18 & 35.07 & Atractylenolide I isomer & $\mathrm{C}_{15} \mathrm{H}_{18} \mathrm{O}_{2}$ & $8.2226 E+08$ & $1.4781 E+09$ & $1.0831 E+09$ & $3.2083 E+09$ \\
\hline 19 & 35.94 & Atractylenolide I & $\mathrm{C}_{15} \mathrm{H}_{18} \mathrm{O}_{2}$ & $8.8877 E+09$ & $7.2520 E+09$ & $8.3857 E+09$ & $1.2742 E+10$ \\
\hline 20 & 39.03 & $\begin{array}{l}\text { 12-methylbutyryl-14-acetyl- } \\
2 \mathrm{E}, 8 \mathrm{EZ}, 10 \mathrm{E} \text {-atractylentriol } \\
\text { isomer I }\end{array}$ & $\mathrm{C}_{21} \mathrm{H}_{26} \mathrm{O}_{5}$ & $3.0978 E+07$ & $3.7863 E+07$ & $2.9171 E+07$ & - \\
\hline 21 & 39.81 & Dibutyl phthalate & $\mathrm{C}_{16} \mathrm{H}_{22} \mathrm{O}_{4}$ & $1.1372 E+08$ & $9.8325 E+07$ & $1.2659 E+08$ & $1.4865 E+08$ \\
\hline 22 & 40.00 & $\begin{array}{l}\text { 12-methylbutyryl-14-acetyl } \\
2 \mathrm{E}, 8 \mathrm{EZ}, 10 \mathrm{E} \text {-atractylentriol } \\
\text { isomer II }\end{array}$ & $\mathrm{C}_{21} \mathrm{H}_{26} \mathrm{O}_{5}$ & $3.8810 E+07$ & $7.7498 E+07$ & $3.3885 E+07$ & $7.0522 E+07$ \\
\hline 23 & 40.26 & Dibutyl phthalate isomer & $\mathrm{C}_{16} \mathrm{H}_{22} \mathrm{O}_{4}$ & $1.0631 E+08$ & $5.4902 E+07$ & $6.1958 E+07$ & $4.6227 E+07$ \\
\hline 24 & 41.50 & $\begin{array}{l}\text { 14-methylbutyryl- } \\
\text { 2E,8EZ,10Es-atractylentriol }\end{array}$ & $\mathrm{C}_{19} \mathrm{H}_{24} \mathrm{O}_{4}$ & $4.9587 E+07$ & $2.8423 E+07$ & $5.1146 E+07$ & $4.7855 E+07$ \\
\hline 25 & 46.43 & Spinasteryl & $\mathrm{C}_{29} \mathrm{H}_{48} \mathrm{O}$ & $8.6778 E+06$ & $7.9096 E+06$ & $1.0609 E+07$ & $7.7832 E+06$ \\
\hline 26 & 47.32 & Atractylon & $\mathrm{C}_{15} \mathrm{H}_{20} \mathrm{O}$ & $7.4433 E+07$ & $5.4063 E+07$ & $6.6146 E+07$ & - \\
\hline 27 & 47.37 & Biatractylolide & $\mathrm{C}_{30} \mathrm{H}_{38} \mathrm{O}_{4}$ & $1.0949 E+09$ & $9.5665 E+08$ & $1.2797 E+09$ & - \\
\hline 28 & 47.96 & Linoleic acid & $\mathrm{C}_{18} \mathrm{H}_{32} \mathrm{O}_{2}$ & $1.8499 E+08$ & $1.5041 E+08$ & $1.8777 E+08$ & $2.3743 E+08$ \\
\hline 29 & 48.25 & Linoleic acid isomer & $\mathrm{C}_{18} \mathrm{H}_{32} \mathrm{O}_{2}$ & $2.1059 E+07$ & - & - & - \\
\hline 30 & 48.59 & Biepiasterolid isomer & $\mathrm{C}_{30} \mathrm{H}_{38} \mathrm{O}_{4}$ & $9.0255 E+08$ & $7.0863 E+08$ & $7.4011 E+08$ & - \\
\hline 31 & 48.90 & Atractylon isomer & $\mathrm{C}_{15} \mathrm{H}_{20} \mathrm{O}$ & $9.5308 E+07$ & $8.7683 E+07$ & $8.2967 E+07$ & $1.0132 E+08$ \\
\hline 32 & 49.42 & Palmitic acid & $\mathrm{C}_{16} \mathrm{H}_{32} \mathrm{O}_{2}$ & $2.2356 E+07$ & $2.2942 E+07$ & $2.5949 E+07$ & $2.0153 E+07$ \\
\hline
\end{tabular}


RT: 0.38-55.95 SM: 3G



(a)

RT: $0.38-55.95$ SM: $3 G$



NL:

$6.97 E 5$

UV_VIS_2 UV2

Z-baishao 1

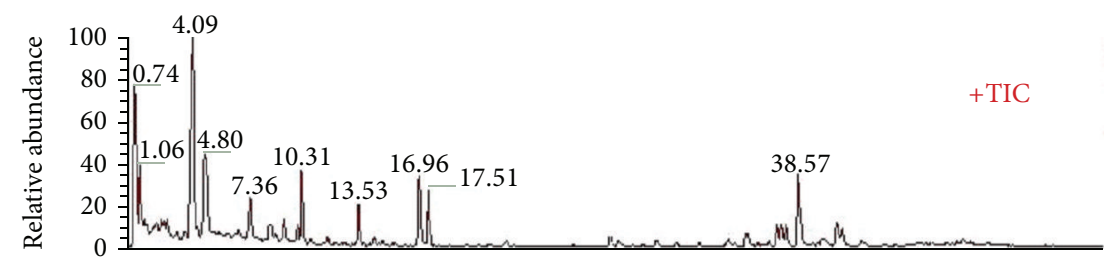

$\mathrm{NL}$ :

5.62E9

TIC F: FTMS + p

ESI Full ms

[150.00-1500.00]

MS Z-baisho 1



NL:

$5.95 E 9$

TIC F: FTMS - p

ESI Full ms

[150.00-1500.00]

MS Z-baisho 1

(b)

FIgURE 1: Total ion chromatograms of crude (a) and processed (b) Paeoniae Radix Alba obtained from both positive and negative ion modes.

\section{Experimental}

2.1. Chemicals, Solvents, and Herbal Materials. Paeoniae Radix Alba and Atractylodis Macrocephalae Rhizoma samples were acquired from Zhejiang suppliers. All of these herbal samples were authenticated by Professor Jianwei Chen (College of Pharmacy, Nanjing University of Chinese Medicine). HPLC-grade acetonitrile and formic acid were obtained from Merck (Darmstadt, Germany). Deionized water was purified using the Milli-Q system (Millipore, Bedford, MA, USA). All other reagents and chemicals were analytical grade.

2.2. Preparation of the Sample Solutions. The dried and powdered samples of crude and processed Paeoniae Radix Alba, crude and processed Atractylodis Macrocephalae Rhizoma, and their crude and processed herbal pair extracts $(1: 1, \mathrm{~g} / \mathrm{g})$ 

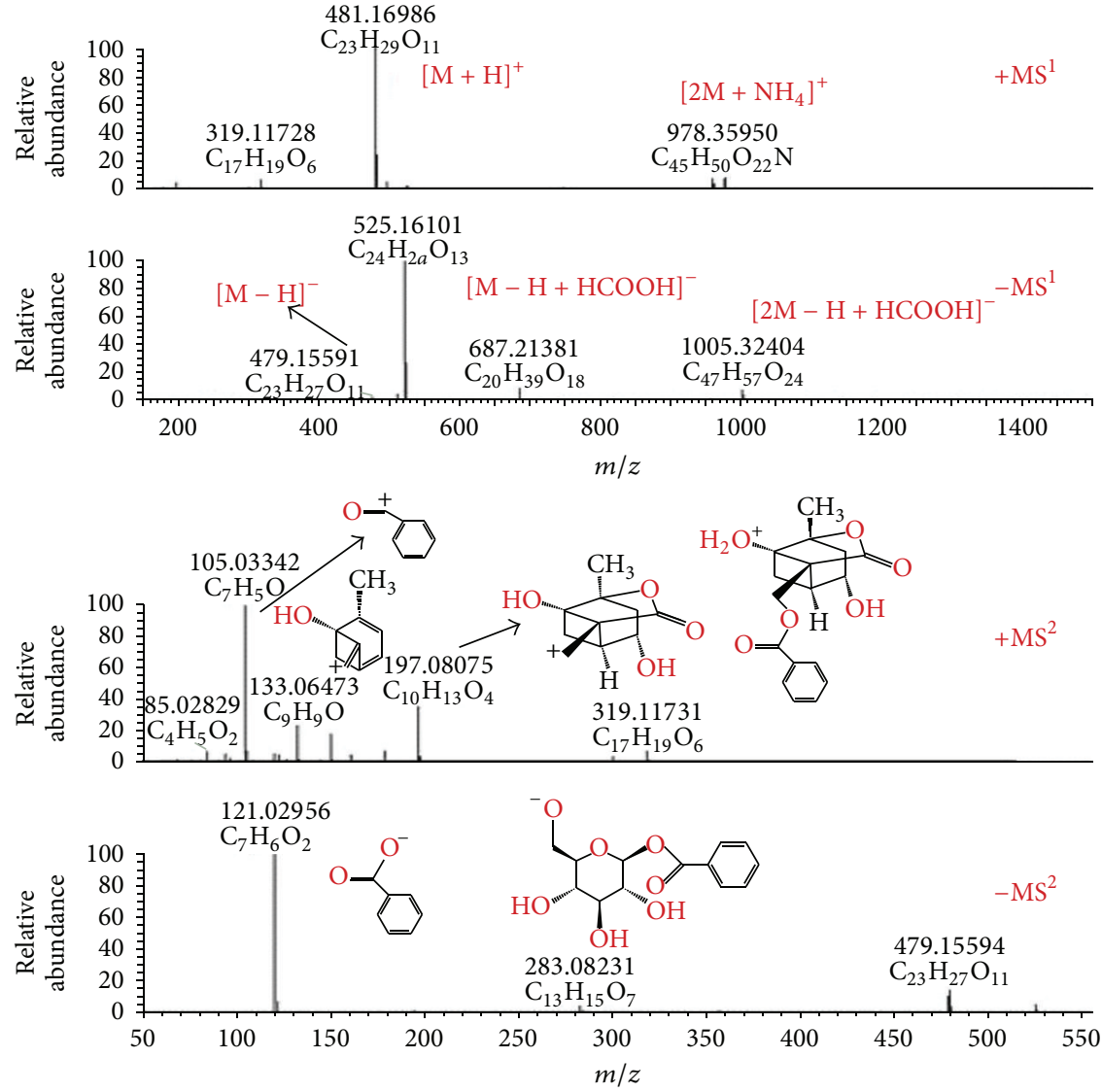

(a)

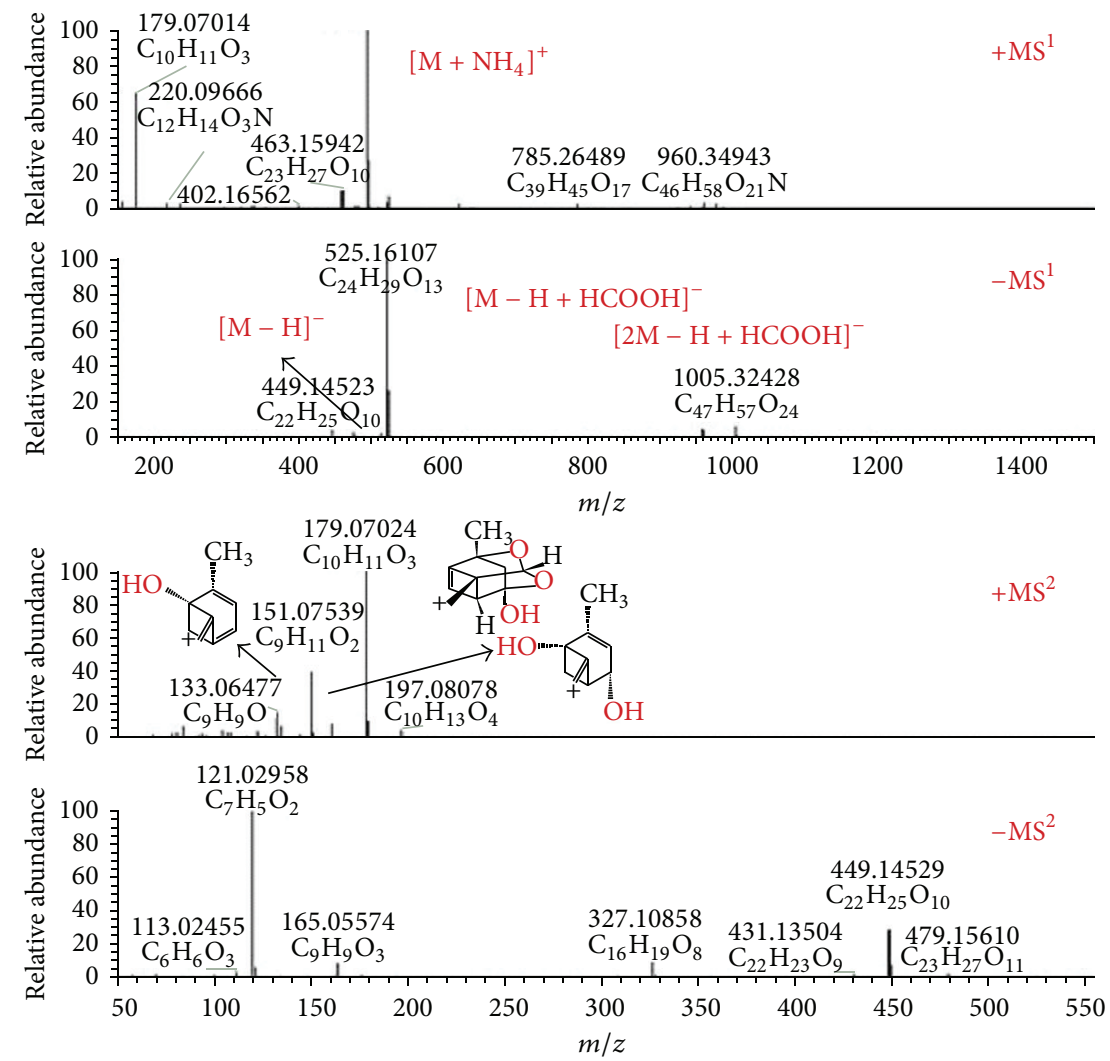

(b)

Figure 2: Continued. 

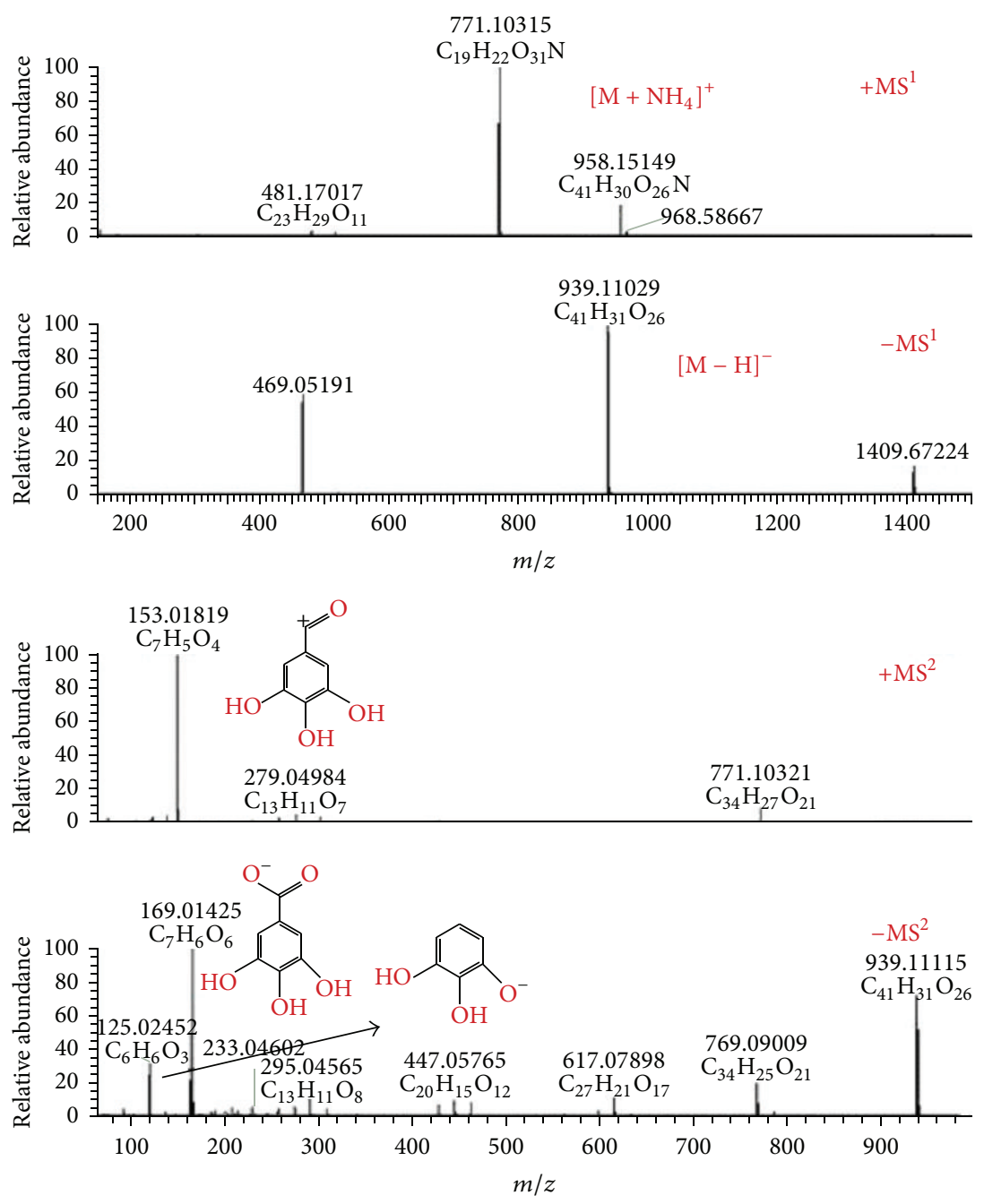

(c)

FIGURE 2: Mass spectra and proposed fragmentations of albiflorin (a), paeoniflorin (b), and 1, 2, 3, 4, 6-penta-O-galloyl-beta-D-glucopyranose (c).

were prepared. A total of $2.0 \mathrm{~g}$ of each sample powder was accurately weighed and transferred into a $50 \mathrm{~mL}$ round bottom flask with $20 \mathrm{~mL}$ of $70 \%$ methanol aqueous solution $(\mathrm{v} / \mathrm{v})$ and refluxed in a $80^{\circ} \mathrm{C}$ water bath for $1 \mathrm{~h}$. The filtrate was collected after filtration and the residue was then refluxed with $20 \mathrm{~mL}$ of $70 \%$ methanol aqueous solution in a $80^{\circ} \mathrm{C}$ water bath for $1 \mathrm{~h}$, the filtrate was collected again after filtration and the residue was removed. Finally, the combined filtrates were treated by rotary evaporation concentration and the resultant residue was dissolved and transferred into a $25 \mathrm{~mL}$ volumetric flask with $70 \%$ methanol aqueous solution to make it up to a final concentration of $0.08 \mathrm{~g} \cdot \mathrm{mL}^{-1}$. All solutions were stored at $4^{\circ} \mathrm{C}$ and filtered through a $0.22 \mu \mathrm{m}$ filter membrane before injection into the HPLC system.

2.3. Liquid Chromatography and Mass Spectrometry. Analyses were performed by using Dionex UltiMate 3000 HPLC system (Dionex, Sunnyvale, CA, USA) with a diode array detector. Detection wavelengths were set at
$255 \mathrm{~nm}$. A Thermo Scientific Hypersil Gold $\mathrm{C}_{18}$ column $(100 \mathrm{~mm} \times 2.1 \mathrm{~mm}, 1.9 \mu \mathrm{m})$ was used with a flow rate of $0.35 \mathrm{~mL} \cdot \mathrm{min}^{-1}$. The injection volume was $5 \mu \mathrm{L}$, and the column temperature was maintained at $30^{\circ} \mathrm{C}$. The sample separation was performed according to the previous reports with minor modification [16-18]. The mobile phase was composed of (a) aqueous formic acid (0.1\%, v/v) and (b) acetonitrile under following gradient elution: $10-55 \% \mathrm{~B}$ from 0 to $40 \mathrm{~min}, 55-90 \%$ B from 40 to $51 \mathrm{~min}, 90 \%$ B from 51 to $56 \mathrm{~min}, 90-10 \% \mathrm{~B}$ from 56 to $56.1 \mathrm{~min}$, and $10 \% \mathrm{~B}$ from 56.1 to $60 \mathrm{~min}$. Mass spectrometry was performed on a $\mathrm{Q}$ Exactive high-resolution benchtop quadrupole Orbitrap mass spectrometer (Thermo Fisher Scientific, San Jose, USA) using a heated electrospray ionization (HESI-II) source for ionization of the target compounds in positive and negative ion modes. The key parameters were as follows: ionization voltage, $+3.0 \mathrm{kV} /-2.8 \mathrm{kV}$; sheath gas pressure, 35 arbitrary units; auxiliary gas, 10 arbitrary units; heat temperature, $300^{\circ} \mathrm{C}$; and capillary temperature, $300^{\circ} \mathrm{C}$. For 
RT: 0.38-55.95 SM: $3 \mathrm{G}$

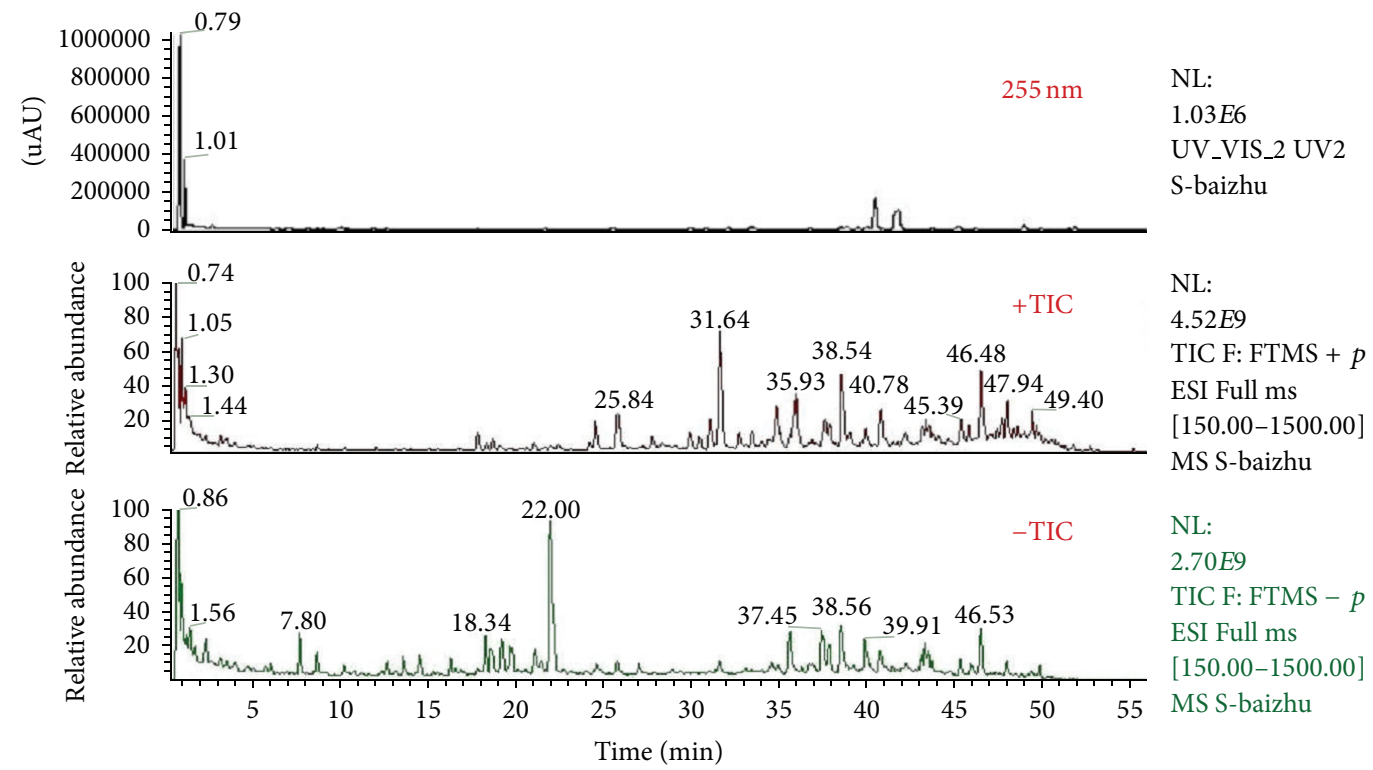

(a)

RT: $0.38-55.95$ SM: $3 G$

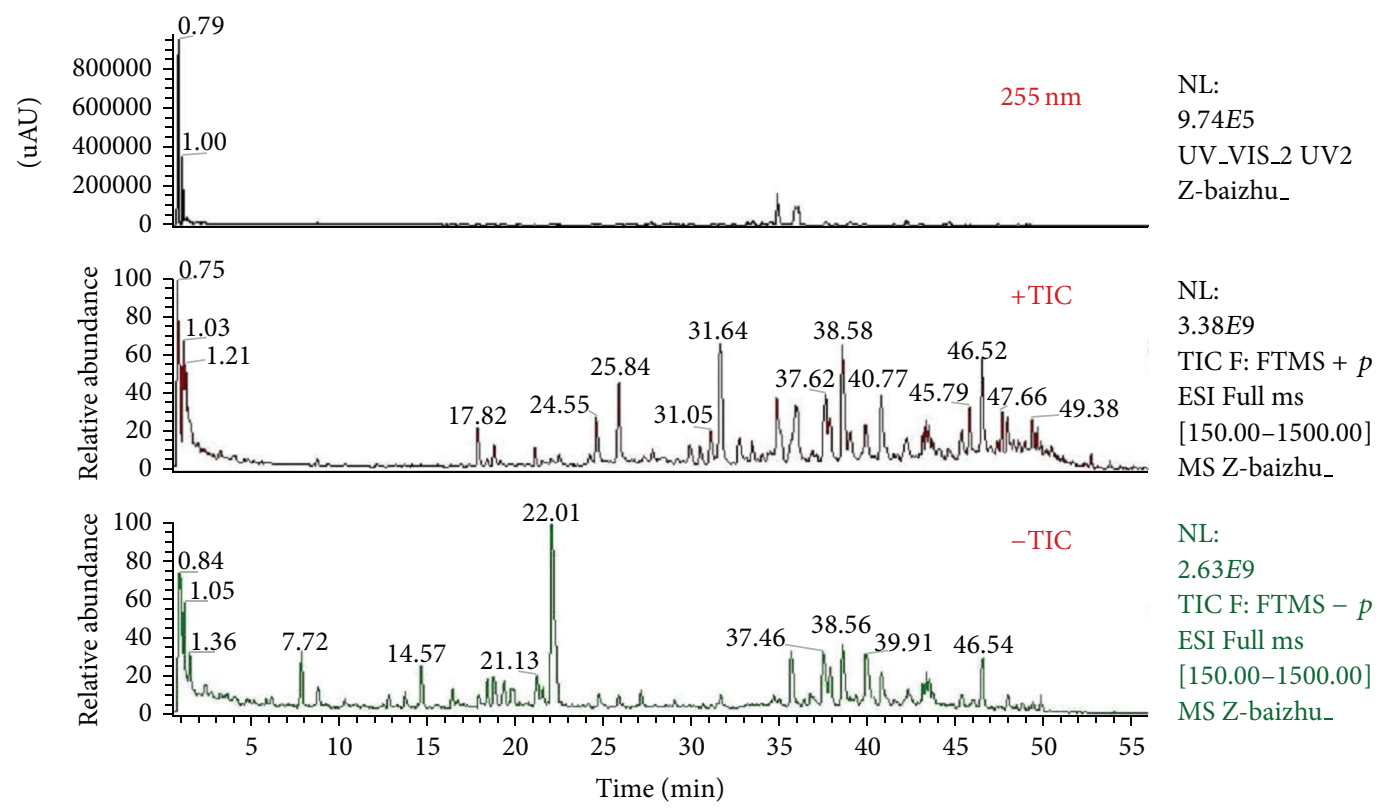

(b)

Figure 3: Total ion chromatograms of crude (a) and processed (b) Atractylodis Macrocephalae Rhizoma obtained from both positive and negative ion modes.

the compounds of interest, a scan range of $m / z 150-1500$ was chosen. Resolution for higher energy collisional dissociation cell (HCD) spectra was set to 17,500 at $\mathrm{m} / z 150$ on the Q Exactive.

\section{Results and Discussion}

3.1. Identification of the Main Components in Crude and Processed Paeoniae Radix Alba. Tentative identification of the main compounds in crude and processed Paeoniae Radix Alba samples was generated based on elemental composition data determined from accurate mass measurements and comparison with the literature data. The total ion chromatograms of crude and processed Paeoniae Radix Alba samples obtained from both positive and negative ion modes were shown in Figure 1. In the preliminary study, the Q Exactive mass spectrometer was confirmed to be highly selective and sensitive. Under the present chromatographic and MS 




(a)

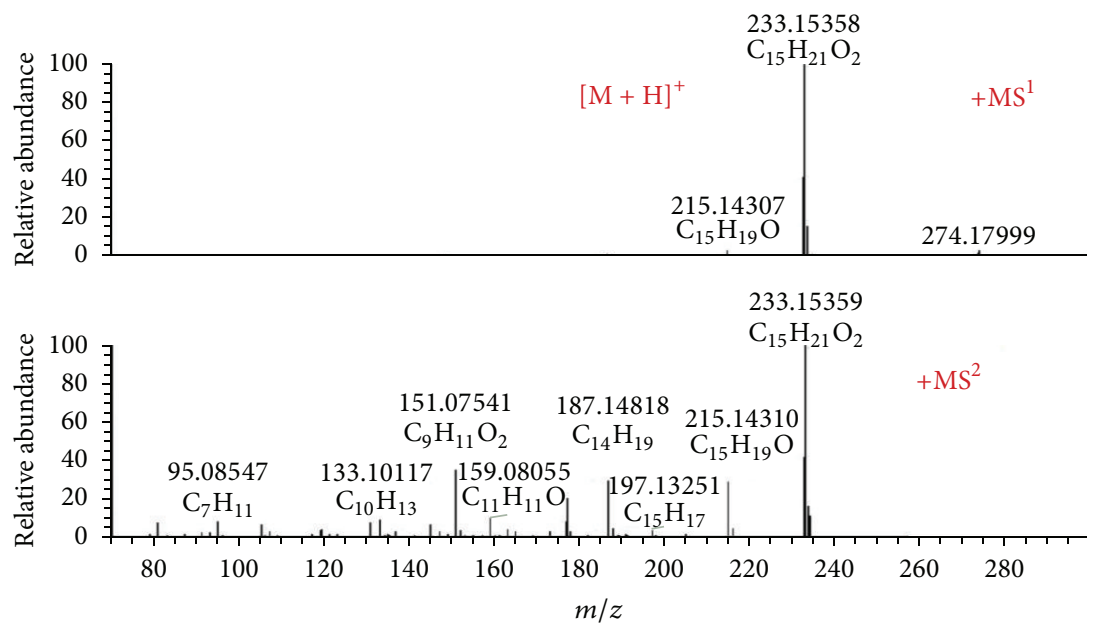

(b)

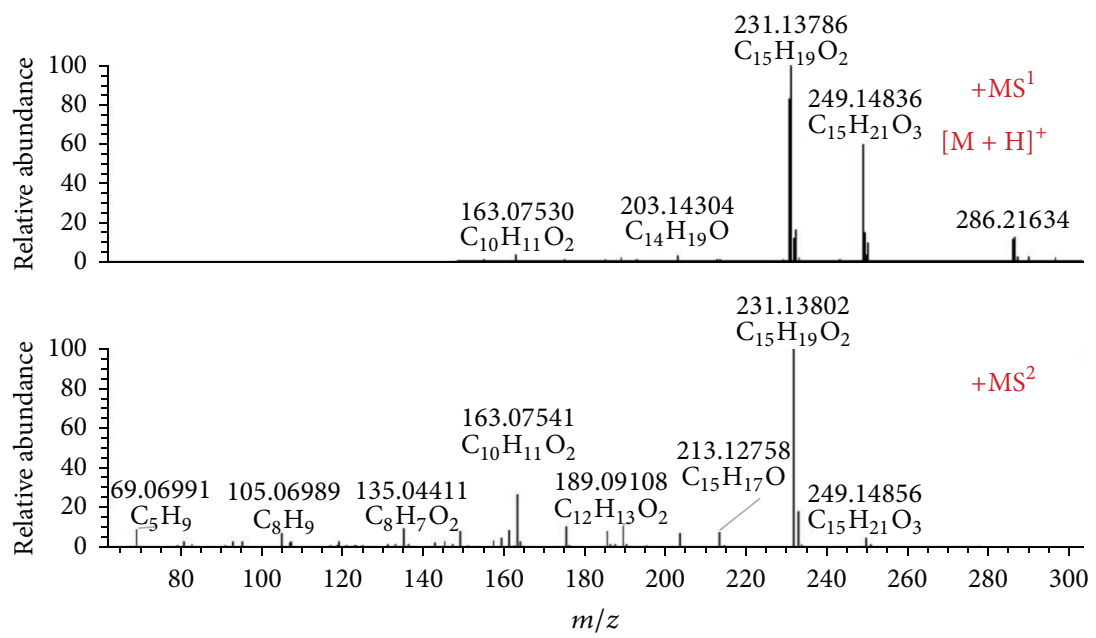

(c)

FIgURE 4: Mass spectra of atractylenolide I (a), atractylenolide II (b), and atractylenolide III (c). 
RT: $0.38-55.95$ SM: $3 G$

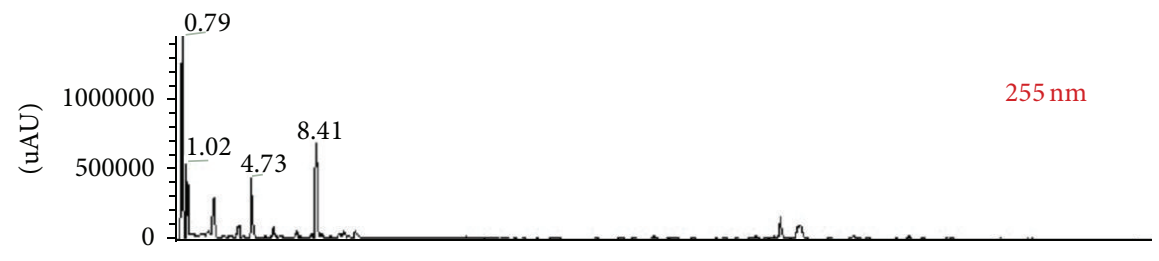

NL:

$1.47 E 6$

UV_VIS_2 UV2

S-peiwu

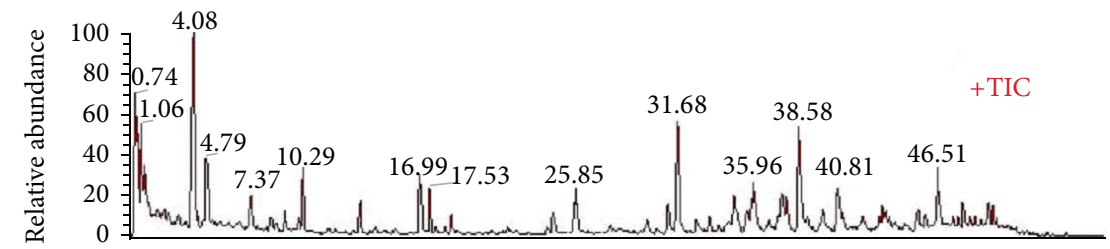

$\mathrm{NL}$ :

6.28E9

TIC F: FTMS + $p$

ESI Full ms

[150.00-1500.00]

MS S-peiwu

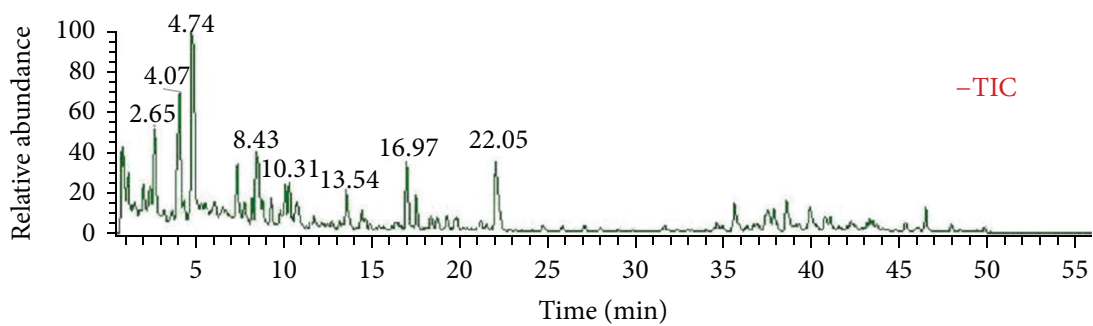

$\mathrm{NL}$

6.93E9

TIC F: FTMS $-p$

ESI Full ms

[150.00-1500.00]

MS S-peiwu

(a)

RT: $0.38-55.95$ SM: $3 G$

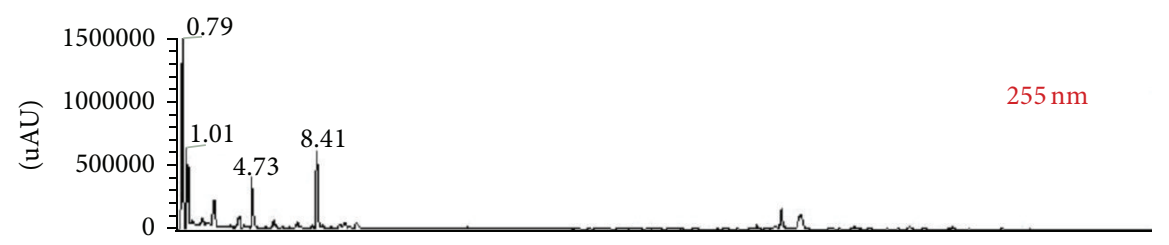

NL:

$1.50 E 6$

UV_VIS_2 UV2

Z-peiwu

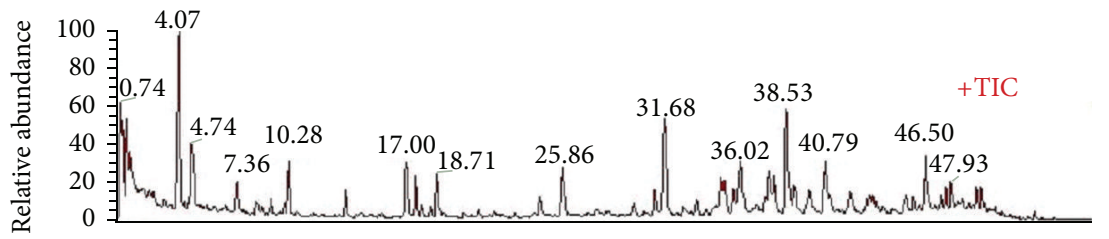

NL:

6.14E9

TIC F: FTMS + $p$

ESI Full ms

[150.00-1500.00]

MS Z-peiwu

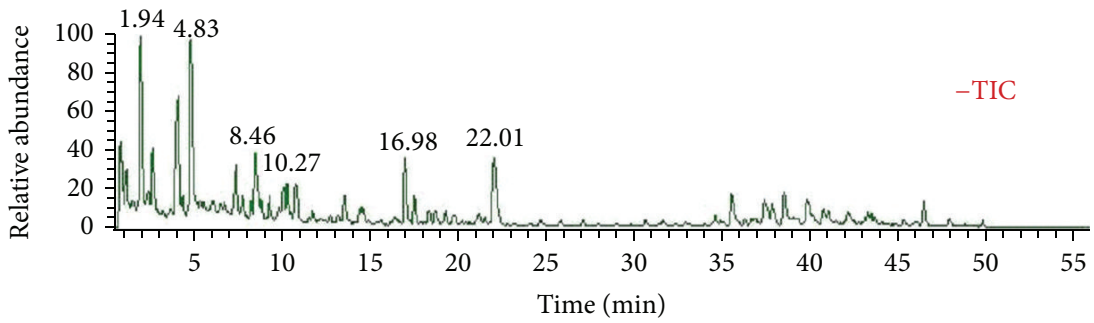

$7.21 E 9$

TIC F: FTMS $-p$

ESI Full ms

[150.00-1500.00]

MS Z-peiwu

(b)

Figure 5: Total ion chromatograms of crude (a) and processed (b) Paeoniae Radix Alba-Atractylodis Macrocephalae Rhizoma herbal pair obtained from both positive and negative ion modes.

conditions, 123 and 101 compounds were identified in crude and processed Paeoniae Radix Alba samples, respectively. Compounds 16, 30, 31, 42, 45, 58, 59, 61, 62, 63, 64, 75, 78, $80,87,90,91,94,95,103,112$, and 120 were not detected in processed Paeoniae Radix Alba sample. Meanwhile, the ESI-MS data of crude and processed samples demonstrated that the peak areas of components 8,113 , and 122 varied significantly, and their amounts were dramatically increased in processed sample. The results were shown in Table 1.

From ESI-MS information, it was found that the sensitivities for all kinds of components in Paeoniae Radix Alba were high in both positive and negative ion modes. 
In present study, we chose peaks 1,2 , and 3 to explain the identification process using $\mathrm{Q}$ Exactive high-performance benchtop quadrupole-Orbitrap LC-MS/MS. Peaks 1, 2, and 3 were eluted at retention times of $4.08,4.79$, and $8.47 \mathrm{~min}$, respectively. Peak 1 showed the $[\mathrm{M}+\mathrm{H}]^{+} \mathrm{m} / z$ 481.16986, $\left[2 \mathrm{M}^{2} \mathrm{NH}_{4}\right]^{+} m / z$ 978.35950, $[\mathrm{M}-\mathrm{H}]^{-} \mathrm{m} / z$ 479.15591, [M$\mathrm{H}+\mathrm{HCOOH}]^{-} m / z$ 525.16101, and $[2 \mathrm{M}-\mathrm{H}+\mathrm{HCOOH}]^{-} \mathrm{m} / z$ 1005.32404 and the corresponding elemental compositions were $\mathrm{C}_{23} \mathrm{H}_{29} \mathrm{O}_{11}, \mathrm{C}_{46} \mathrm{H}_{60} \mathrm{O}_{22} \mathrm{~N}, \mathrm{C}_{23} \mathrm{H}_{27} \mathrm{O}_{11}, \mathrm{C}_{24} \mathrm{H}_{29} \mathrm{O}_{13}$, and $\mathrm{C}_{47} \mathrm{H}_{57} \mathrm{O}_{24}$, respectively. On the basis of above data we deduced that the elemental composition of peak 1 was $\mathrm{C}_{23} \mathrm{H}_{28} \mathrm{O}_{11}$. The molecular ion of peak 1 could lead to seven main $\mathrm{MS}^{2}$ ions at $m / z$ 319.11731, 197.08075, 133.06473, and 105.03342 in positive ion mode, and $m / z$ 479.15594, 283.08231, and 121.02956 in negative ion mode. On the basis of the elemental compositions of fragment ions, peak 1 was assigned as albiflorin. Peaks 2 and 3 were therefore identified as paeoniflorin, and 1, 2, 3, 4, 6-penta-O-galloyl-beta-Dglucopyranose with above mentioned method. The mass spectra and proposed fragmentations of albiflorin, paeoniflorin, and 1, 2, 3, 4, 6-penta-O-galloyl-beta-D-glucopyranose were shown in Figure 2.

\subsection{Identification of the Main Components in Crude and Pro-} cessed Atractylodis Macrocephalae Rhizoma. Figure 3 showed the total ion chromatograms of crude and processed Atractylodis Macrocephalae Rhizoma samples obtained from both positive and negative ion modes. 32 and 26 compounds were identified in crude and processed Atractylodis Macrocephalae Rhizoma samples, respectively. Compounds 2, 4, 13, 14,17 , and 29 were not detected in processed Atractylodis Macrocephalae Rhizoma sample. Moreover, the amounts of compounds $3,7,9,10,21,23$, and 27 were substantially decreased, and the amounts of compounds 8,18 , and 22 were increased in processed sample compared with crude one. The results were shown in Table 2.

Atractylenolide I, atractylenolide II, and atractylenolide III are the main active compounds that belong to the sesquiterpenes in Atractylodis Macrocephalae Rhizoma. The mass spectra of atractylenolide I showed a $[\mathrm{M}+\mathrm{H}]^{+}$ion at $\mathrm{m} / \mathrm{z}$ 231.13799, which could lead to four $\mathrm{MS}^{2}$ ions at $m / z 213.12740$, 185.13251, 157.10127, and 143.08569. The molecular ion of atractylenolide II $\left([\mathrm{M}+\mathrm{H}]^{+} \mathrm{m} / z\right.$ 233.15358) could lead to six $\mathrm{MS}^{2}$ ions at $\mathrm{m} / z$ 215.14310, 187.14818, 159.08055, 151.07541, 133.10117, and 95.08547. Meanwhile, the $\mathrm{MS}^{2}$ spectrum of $\mathrm{m} / z$ 249.14836 from atractylenolide III contained six major fragment ions at $\mathrm{m} / z$ 231.13802, 213.12758, 189.09108, 163.07541, 135.04411 , and 105.06989. The mass spectra of the above three compounds were shown in Figure 4.

3.3. Analysis of Chemical Changes of Paeoniae Radix Alba after Compatibility with Atractylodis Macrocephalae Rhizoma. In the present study, the Q Exactive high-performance benchtop quadrupole-Orbitrap LC-MS/MS based on chemical profiling approach was used to evaluate chemical constitution between co-decoction and single decoction of Paeoniae Radix Alba and Atractylodis Macrocephalae Rhizoma. For crude Paeoniae Radix Alba, the relative contents of most compounds were dramatically decreased except those of compounds $80,90,98,113,119$, and 122 were significantly increased and 19 compounds were not detected after its compatibility with crude Atractylodis Macrocephalae Rhizoma. For processed Paeoniae Radix Alba, the relative contents of compounds 12, 36, 84, and 86 were remarkably increased except 12 compounds including pedunculagin, oxypaeoniflorin, 6-O-glucopyranosyl-lactinolide, 1, 2, 3, 6-tetra-Ogalloylglucose isomer $\mathrm{A}, 1,2$, 3, 6-tetra-O-galloylglucose isomer $\mathrm{B}$, tetragalloyl glucose $\mathrm{C}$, galloylpaeoniflorin isomer II, hexagalloyl glucose, 3, 6-di-O-galloyl paeoniorin isomer, oxybenzoyl-oxypaeoniflorin, benzoyloxypaeoniflorin, and albiflorin R1 isomer III were newly generated and 13 compounds were not found after its compatibility with processed Atractylodis Macrocephalae Rhizoma. The results were presented in Figure 5 and Table 1.

3.4. Analysis of the Chemical Changes of Atractylodis Macrocephalae Rhizoma after Compatibility with Paeoniae Radix Alba. For crude Atractylodis Macrocephalae Rhizoma, the relative contents of compounds 17,18 , and 25 were increased clearly except those of compounds 6, 23, and 30 decreased considerably and six compounds including protocatechuic acid isomer I, protocatechuic acid isomer II, atracetylentriol, 12-methylbutyryl-14-acetyl-2E, 8EZ, 10E-atractylentriol, 12methylbutyryl-14-acetyl-2E, 8EZ, 10E-atractylentriol isomer, and linoleic acid isomer were lost after its compatibility with crude Paeoniae Radix Alba. For processed Atractylodis Macrocephalae Rhizoma, compounds 9, 20, 26, 27, and 30 were not found except the relative contents of compounds 5,6 , and 8 were decreased while those of compounds 15 , 19, 21, and 31 were increased after its compatibility with processed Paeoniae Radix Alba. Furthermore, compound 4 (protocatechuic acid isomer II) was not found in processed Atractylodis Macrocephalae Rhizoma but could be detected in processed Paeoniae Radix Alba-Atractylodis Macrocephalae Rhizoma herbal pair by using Exact Finder and MassFrontier softwares. The above results illustrated that Paeoniae Radix Alba significantly changed the components of Atractylodis Macrocephalae Rhizoma in solution when they decocted together. The corresponding results were presented in Figure 5 and Table 2.

\section{Conclusions}

Q Exactive high-performance benchtop quadrupoleOrbitrap LC-MS/MS is a powerful tool for discriminating the chemical changes between single herbal and codecocting medicines. In our present study, the $\mathrm{Q}$ Exactive high-performance benchtop quadrupole-Orbitrap LCMS/MS based on chemical profiling approach to investigate and evaluate chemical changes from crude and processed Paeoniae Radix Alba, crude and processed Atractylodis Macrocephalae Rhizoma, and their crude and processed herbal pair extracts was proposed. The results showed that processing and compatibility of TCM could significantly change the chemical composition of Paeoniae Radix Alba and Atractylodis Macrocephalae Rhizoma. The developed 
method is considered to provide a scientific foundation for deeply elucidating the processing and compatibility mechanism of Paeoniae Radix Alba and Atractylodis Macrocephalae Rhizoma.

\section{Conflict of Interests}

The authors declare that there is no conflict of interests regarding the publication of this paper.

\section{Authors' Contribution}

Gang Cao, Qinglin Li, Hao Cai, and Sicong Tu contributed equally to this work.

\section{Acknowledgments}

This work was financially supported by the National Natural Science Foundation of China (nos. 81202918, 81173546, and 30940093), the Natural Science Foundation of Jiangsu Province, China (no. BK2009495), the International Science and Technology Cooperation Project of Jiangsu Province, China (no. BZ2011053), the Open Project of National FirstClass Key Discipline for Science of Chinese Materia Medica, Nanjing University of Chinese Medicine (no. 2011ZYX2006), the Project of Science and Technology for Chinese Medicine of Zhejiang Province, China (no. 2013KYB183), the Science and Technology Project of Hangzhou, China (nos. 20130533B68, 20131813A23), the Chinese Medicine Research Program of Zhejiang Province, China (nos. 2014ZQ008, 2008ZA002), the Project of Science Technology Department of Zhejiang Province, China (no. 2013C33SA1C0003), and the Science Foundation of Zhejiang Chinese Medical University (nos. 2011ZY25, 2013ZZ12).

\section{References}

[1] K. M. Qin, Y. C. Shu, G. Cao et al., "Thoughts and methods of Chinese materia medica processing-taking research on Rehmanniae Radix processing as an example," Chinese Traditional and Herbal Drugs, vol. 44, no. 11, pp. 1363-1370, 2013.

[2] K. M. Qin, L. J. Zheng, G. Cao et al., "Ideas and methods for mechanism research of traditional Chinese medicine processing - taking coffee beans roasting mechanism research as an example," Scientia Sinica Chimica, vol. 43, no. 7, pp. 829839, 2013.

[3] S. L. Li, S. F. Lai, J. Z. Song et al., "Decocting-induced chemical transformations and global quality of Du-Shen-Tang, the decoction of ginseng evaluated by UPLC-Q-TOF-MS/MS based chemical profiling approach," Journal of Pharmaceutical and Biomedical Analysis, vol. 53, no. 4, pp. 946-957, 2010.

[4] C. Wang, Y. G. Wang, Q. D. Liang, W. Q. Rang, C. R. Xiao, and Y. Gao, "Analysis of chemical composition in combination of Aconitum and Fritillaria by UPLC/Q-TOFMS with multivariate statistical analysis," Acta Chimica Sinica, vol. 69, no. 16, pp. 19201928, 2011.

[5] Z. C. Ma, S. S. Zhou, Q. D. Liang et al., "UPLC-TOF/MS based chemical profiling approach to evaluate toxicity-attenuated chemical composition in combination of ginseng and Radix
Aconiti Praeparata," Acta Pharmaceutica Sinica, vol. 46, no. 12, pp. 1488-1492, 2011.

[6] W. W. Peng, S. S. Liu, Y. Wang et al., "Effect of Compatibility of Radix Aconiti Lateralis Praeparate and Zingiber offiicinale Bosc. on the contents of four aconitum alkaloids," Chinese Pharmaceutical Journal, vol. 48, no. 4, pp. 258-261, 2013.

[7] L. J. Zheng, K. M. Qin, H. Cai, G. Cao, and B. C. Cai, "Optimization of extraction process for Baizhu Shaoyao San by multi-index orthogonal experiment," China Journal of Chinese Materia Medica, vol. 38, no. 10, pp. 1504-1509, 2013.

[8] X. C. Li, J. Lin, W. J. Han et al., "Antioxidant ability and mechanism of Rhizoma Atractylodes macrocephala," Molecules, vol. 17, no. 11, pp. 13457-13472, 2012.

[9] L. L. Yu, T. Z. Jia, and Q. Cai, "Research on the chemical references of Atractylodes Macrocephala Koidz," Asia-Pacific Traditional Medicine, vol. 6, no. 3, pp. 36-39, 2010.

[10] H. Cai, Z. W. Xu, S. C. Luo et al., "Study on chemical fingerprinting of crude and processed Atractylodes macrocephala from different locations in Zhejiang province by reversedphase high-performance liquid chromatography coupled with hierarchical cluster analysis," Pharmacognosy Magazine, vol. 8, no. 32, pp. 300-307, 2012.

[11] S. H. Rong, H. Lin, and N. Gao, "Study on processing technology and processing principles of Atractrylodis Macrocephalae Rhizoma," China Journal of Chinese Materia Medica, vol. 36, no. 8, pp. 1001-1003, 2011.

[12] L. Yang, X. H. Xia, Q. Zhu, and X. P. Tan, "Study on the rule of influence of two purification methods on the chemical compositions in aqueous solution of Paeoniae Radix Alba," Journal of Chinese Medicinal Materials, vol. 36, no. 1, pp. 118121, 2013.

[13] N. C. Luo, W. Ding, J. Wu et al., "UPLC-Q-TOF/MS coupled with multivariate statistical analysis as a powerful technique for rapidly exploring potential chemical markers to differentiate between radix paeoniae alba and radix paeoniae rubra," Natural Product Communications, vol. 8, no. 4, pp. 487-491, 2013.

[14] S. J. Huang, R. Wang, Y. H. Shi, L. Yang, Z. Y. Wang, and Z. T. Wang, "Primary safety evaluation of sulfated Paeoniae Radix Alba," Acta Pharmaceutica Sinica, vol. 47, no. 4, pp. 486-491, 2012.

[15] K. B. Kwon, E. K. Kim, M. J. Han et al., "Induction of apoptosis by Radix Paeoniae Alba extract through cytochrome c release and the activations of caspase- 9 and caspase- 3 in HL-60 cells," Biological and Pharmaceutical Bulletin, vol. 29, no. 6, pp. 10821086, 2006.

[16] Z. Q. Yu, R. M. Schmaltz, T. C. Bozeman et al., "Selective tumor cell targeting by the disaccharide moiety of bleomycin," Journal of the American Chemical Society, vol. 135, no. 8, pp. 2883-2886, 2013.

[17] T. Kabashima, Z. Q. Yu, C. H. Tang et al., "A selective fluorescence reaction for peptides and chromatographic analysis," Peptides, vol. 29, no. 3, pp. 356-363, 2008.

[18] J. Y. Pan and Z. Q. Yu, "Isolation and characterization of Hainantoxin-II, a new neurotoxic peptide from the Chinese bird spider (Haplopelma hainanum)," Zoological Research, vol. 31, no. 6, pp. 570-574, 2010. 




The Scientific World Journal
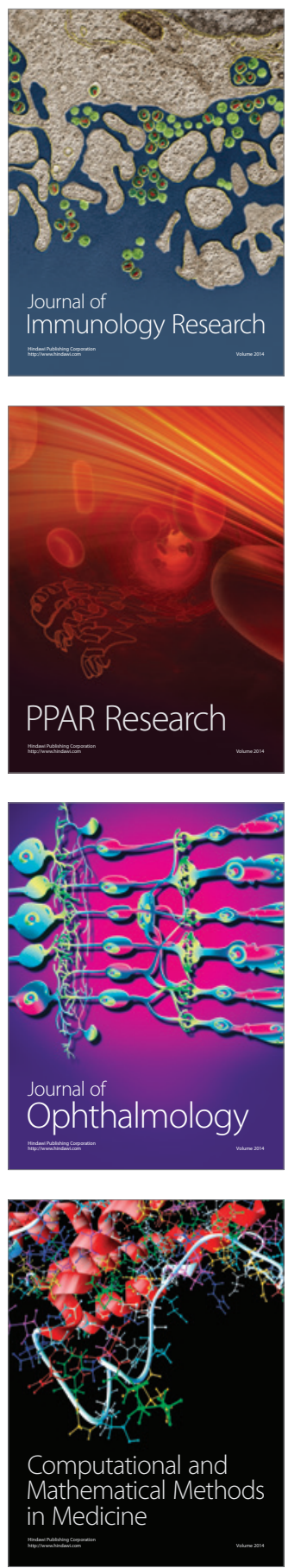



Gastroenterology

Research and Practice
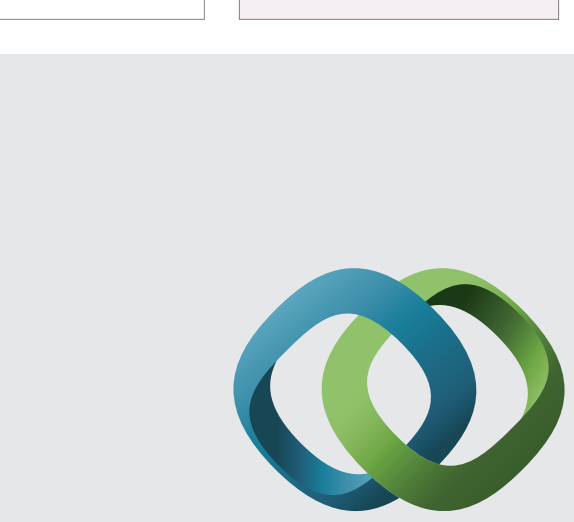

\section{Hindawi}

Submit your manuscripts at

http://www.hindawi.com
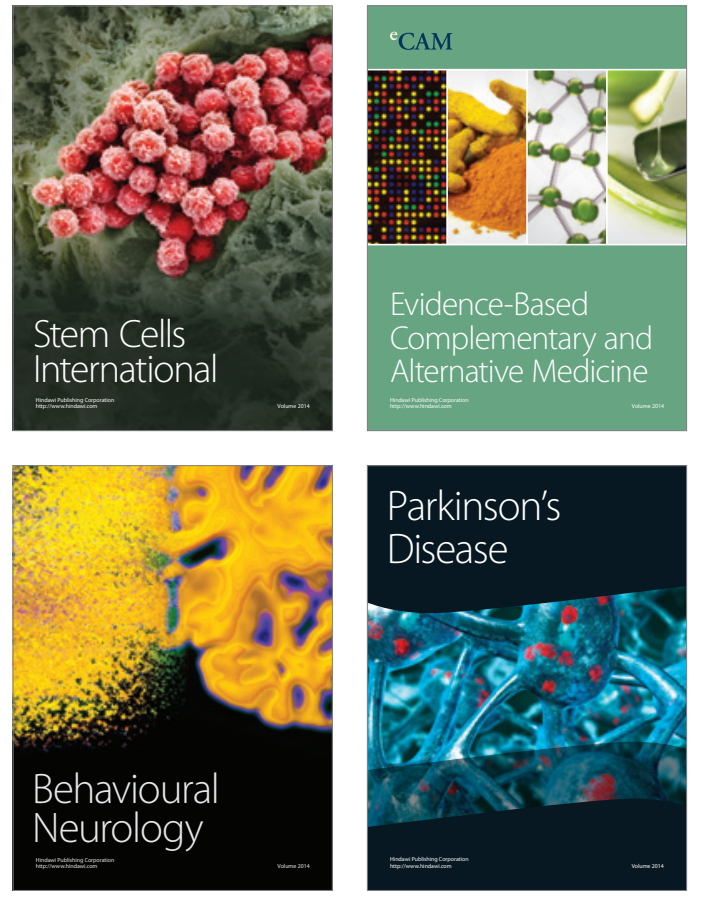
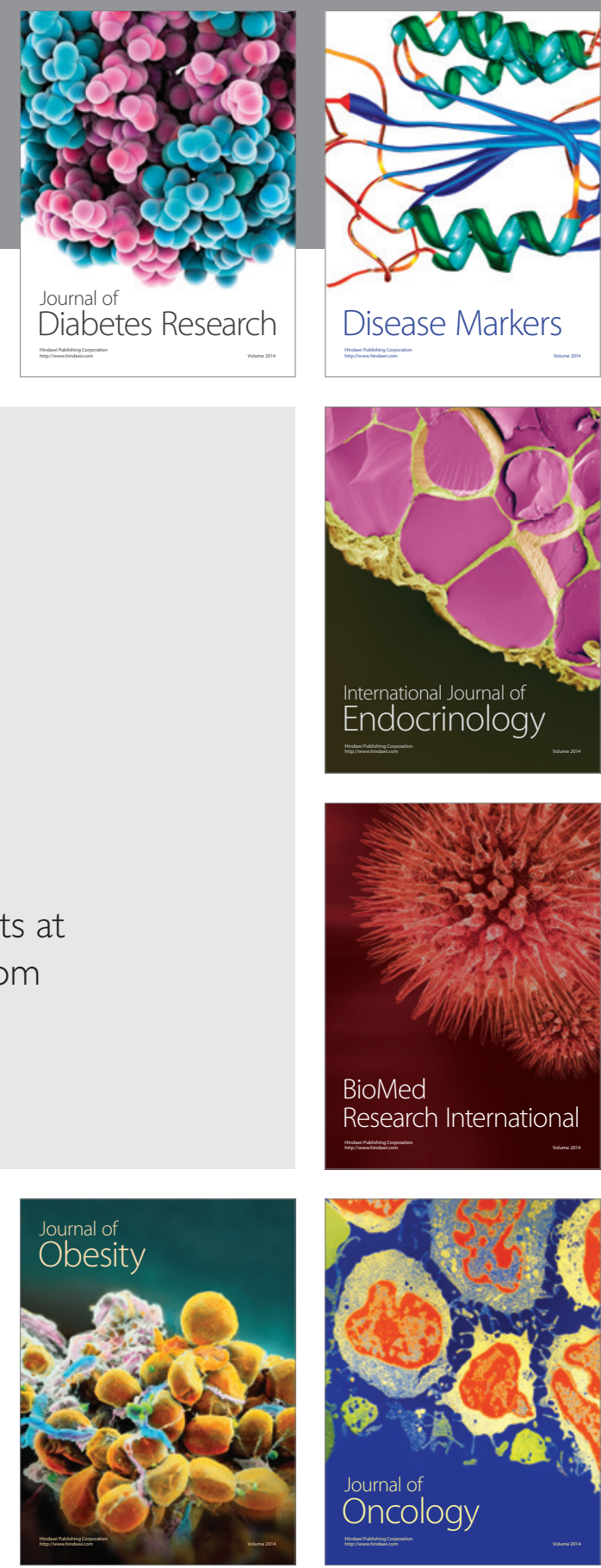

Disease Markers
